\title{
Revisiting Java Bytecode Compression for Embedded and Mobile Computing Environments
}

\author{
Dimitris Saougkos, George Manis, Konstantinos Blekas, Member IEEE, Apostolos V. Zarras, Member IEEE
}

\begin{abstract}
Pattern-based Java bytecode compression techniques rely on the identification of identical instruction sequences that occur more than once. Each occurrence of such a sequence is substituted by a single instruction. The sequence defines a pattern that is used for extending the standard bytecode instruction set with the instruction that substitutes the pattern occurrences in the original bytecode. Alternatively, the pattern may be stored in a dictionary that serves for the bytecode decompression. In this case, the instruction that substitutes the pattern in the original bytecode serves as an index to the dictionary. In this paper, we investigate a bytecode compression technique that considers a more general case of patterns. Specifically, we employ the use of an advanced pattern discovery technique that allows locating patterns of an arbitrary length, which may contain a variable number of wildcards in place of certain instruction opcodes or operands. We evaluate the benefits and the limitations of this technique in various scenarios that aim at compressing the reference implementation of MIDP, a standard Java environment for the development of applications for mobile devices.
\end{abstract}

Index Terms-D.3.2.j Java, I.4.2 Compression (Coding).

\section{INTRODUCTION}

$\mathbf{T}$ HE Java language has become a dominant means for the realization of embedded and mobile computing environments. The main feature of Java that led to the previous is its portability. More specifically, the compilation of Java applications results in device independent code, generated in terms of a standard format, called Java bytecode. The Java bytecode can then execute on top of different devicespecific Java Virtual Machines (JVMs), which take charge of translating the bytecode into device-specific machine code.

The memory limitations imposed by embedded and mobile devices certainly constrain the set of applications that may possibly execute on top of them. Confronting the aforementioned issue fosters research towards two orthogonal directions. The first one concerns the reduction of the physical size and cost of memory chips, while the second one involves reducing the size of the code of embedded applications. Advances in both of the previous research directions are equally valuable. No matter how much we increase the amount of available memory, there will always be more demanding applications. Similarly, even if we manage to diminish the size of embedded and mobile applications we may always require to concurrently execute as many of them as possible.

Lots of significant research efforts have already been done towards the generation of compressed code [1]. However, most of these efforts involve the compression of either machine or

D. Saougkos, G. Manis, K. Blekas and A. V. Zarras are with the Computer Science Department of the University of Ioannina, P.O. BOX 1186 GR 45110 Greece. Email: \{dsaougos, manis, kblekas, zarras\}@cs.uoi.gr assembly code. Amongst the few approaches that focus on the case of Java bytecode we have the ones proposed in [2], [3] and [4]. In [2] the authors examine various approaches for bytecode compression, relying on Huffman codes and Markov chains. In [3], bytecode compression is based on the use of canonical Huffman codes and the generation of fast decoders. In [4], the approach followed is based on the discovery of instruction sequences that occur more than once within the Java bytecode. Each sequence of instructions defines a pattern. Each pattern occurrence is substituted by a single instruction that is called a macro.

However, a pattern, in its broadest sense may have the following characteristics:

1) It may be of an arbitrary length.

2) It may contain wildcards in place of a particular opcode or operand. Hereafter, we use the term parameterized to refer to patterns that contain a variable number of wildcards. Respectively, we use the term non-parameterized to refer to patterns that do not contain wildcards.

So far, existing approaches for Java bytecode compression do not deal with the aforementioned generic form of patterns. This fact is recognized in [4] where the authors further highlight the need for more sophisticated pattern discovery techniques. The main contribution of this paper is to assess the use of such a technique in the context of Java bytecode compression. Specifically:

- We customize a well-known pattern discovery technique, called agglomerative clustering [5], [6], towards the identification of parameterized and non-parameterized patterns within a given Java bytecode. The proposed technique allows discovering patterns of an arbitrary length, which may contain a variable number of wildcards.

- We assess the advantages and the limitations of the aforementioned technique in various scenarios that aim at compressing MIDP, a standard Java environment that supports the development of applications for mobile devices. The main feature of the parameterized pattern discovery technique is that it allows finding a large variety of patterns that can be combined to obtain better bytecode size reduction. This, however, is also its main limitation. Exploring the large variety of patterns towards finding a combination that gives a good bytecode size reduction is a complex task. Given this fact, in our assessment:

1) We employ/compare two heuristic methods towards the combination of patterns.

2) Based on the two heuristics, we investigate the impact of using patterns that contain a variable 
number of wildcards for the compression of Java bytecode. To this end, we compress MIDP using patterns that contain a variable number of wildcards, patterns that do not contain wildcards and patterns that contain a fixed number of wildcards and we perform a comparative study of the results.

3) Moreover, we examine the impact of the increasing length of the patterns in the compression of Java bytecode.

4) Finally, we study the behavior of the decompression overhead introduced by the examined technique.

The remainder of this paper is structured as follows. In Section 2, we discuss related work. In Section 3, we introduce a typical non-parameterized pattern discovery technique, followed by the advanced parameterized pattern discovery technique that we investigate. Following, we highlight the benefits of the parameterized pattern discovery technique, as opposed to the non-parameterized one, with respect to a number of motivating examples. In Section 4, we introduce the complementary heuristic techniques, which serve for handling the complexity of combining large numbers of parameterized and non-parameterized patterns. Moreover, we present the experimental results we obtained in the case of MIDP. Finally, in Section 5 we summarize the contribution of this paper and point out the future directions of this work.

\section{RELATED WORK}

Code compression techniques can be divided into two major categories [7]. The first one aims at producing a reducedsize wire code that can be transmitted to the CPU as fast as possible. In this case, what matters is to achieve the best possible compression. For the particular case of Java, there have been several approaches for the construction of wire code. Amongst the prominent ones we have JAZZ [8], an alternative to the standard JAR format and Slim Binaries [9], an alternative to the standard bytecode format. Moreover, we have the approach proposed in [2], where the authors discuss methods for reducing the size of the constant pool. In [10], Pugh also discusses interesting ideas towards a wire code format that aims at reducing the size of collections of class files. Finally, in [11] the authors investigate techniques for the removal of redundant class file attributes and methods, along with techniques for constant pool compression and class hierarchy transformations.

The second category of techniques aims at producing a reduced-size interpretable code that can be stored and executed without being fully decompressed. In this case, what is important is the reduction of the overall amount of memory required for the execution of the application. The various techniques proposed for the generation of interpretable code rely either on Huffman codes and arithmetic coding, or on the identification of patterns.

The use of Huffman codes [12], [13] and arithmetic coding [14] in code compression aims at shortening a sequence of instructions by mapping them into the shortest possible sequence of bits. Huffman based techniques have been criticized for their decompression complexity. However, in [3] Latendresse and
Feeley propose an approach for fast Huffman decoding. Their approach focuses on virtual instructions and is evaluated for the case of Java bytecode. In [2], the authors also investigate the use of Huffman codes for compressing Java bytecode.

Pattern-based techniques for interpretable code compression focus on the identification of multiple occurrences of instruction sequences within an application. Each such sequence is called a pattern. Patterns are usually stored in a dictionary and their occurrences in the original code are substituted in the compressed code by dictionary indexes. Indexes are frequently called macros and their size is usually the size of a single instruction. Specifically, in [15] the authors propose a dictionary-based approach that can be applied in RISC intermediate representations. This approach allows searching for non-parameterized patterns of an arbitrary length. Nonparameterized patterns of an arbitrary length are also used by the IBM CodePack compressor [16], which is deployed on PowerPCs. This particular technique originates from the one proposed by Lefurgy et al. in [17].

In [18], the authors present an approach that deals with parameterized patterns. The approach relies on the discovery of similar basic blocks (i.e., code fragments with a unique entry and a unique exit point). As a similarity metric the authors use a fingerprint function. Moreover, several classical compiler optimization techniques (e.g., dead code elimination) are applied. In [19], the authors go one step further by searching for similar procedures and code regions. Again, the fingerprint function is used as a similarity metric. Parameterized procedures are also used in Krinke's work [20]. Moreover, in BRISC [7] the authors propose a dictionary-based technique that relies on patterns consisting of 2 instructions. The patterns may further contain wildcards in place of instruction operands. Parameterized pattern discovery is also used in [21]. This approach focuses on the identification of similar basic blocks in ARM code. For a set of similar blocks, a representative function is built. The representative function comprises predicated instructions, corresponding to the differences met amongst the original basic blocks. In [22], the authors also propose an approach that employs a sort of parameterized patterns. The proposed system accepts as input a grammar and a training set of programs and produces an expanded grammar that allows shorter derivations of the training and other similar programs. The rules of the expanded grammar can be seen as parameterized patterns, leading to different derivations. In [20], legacy source code is transformed using procedural abstraction so that it becomes more understandable, maintainable and small. Extracted sets of statements form procedures and the extracted code is replaced with procedure calls. Similarly, in [23] the authors identify similar segments of source code based on program dependence graphs.

Considering the particular case of Java bytecode, in [4] the authors propose a technique which considers nonparameterized patterns of an arbitrary length. Their main contribution is that they do not directly use the notion of a dictionary; instead they specialize the JVM with more complex instructions that actually realize the execution of the patterns. Then, the patterns can be substituted in the original code by the new instructions, reducing thus the size 
of the original code. In this paper, we investigate a dictionarybased bytecode compression technique, which goes one step further from [4] by considering both parameterized and nonparameterized patterns of an arbitrary length. Currently, the technique is applied in bytecode basic blocks but it could be as well used in the case of whole Java methods. The technique that we investigate is more fine-grained with respect to the other parameterized pattern discovery techniques, discussed in this section. It does not search for similarity among whole basic blocks (or procedures). Instead, it searches inside the basic blocks for similar instruction sequences of an arbitrary length. To cope with the increased complexity of this task, we employed the agglomerative clustering algorithm [5], [6].

\section{JAVA B YTECODE COMPRESSION PROCESS}

The bytecode of a compiled Java program is a sequence of binary encoded JVM instructions. Each instruction consists of an opcode and possibly a number of operands. The size of instruction opcodes or operands is 1 byte. As an example, consider the Java program given in Figure 1(a). This simple program comprises a class of objects that represent vectors in the 3-dimensional space. Each vector is characterized by 3 coordinates (x, y, z, attributes) and provides a method, called distance, which calculates the Euclidean distance norm, $d=\sqrt{x^{2}+y^{2}+z^{2}}$, for the vector.

The compiled bytecode for the distance method of our program is given in Figure 1(b). The overall size of the sequence is 34 bytes. In the remainder of this section, we use the example of Figure 1(b) to highlight the main steps of the non-parameterized and the parameterized compression approaches, discussed in this section. In both cases, the overall compression process consists of the following steps:

1) The Java bytecode is segmented into basic blocks.

2) A pattern discovery technique is used for the identification of patterns in the basic blocks of the bytecode.

3) The resulted patterns are collected; possible combinations of patterns are examined and for each one of them the corresponding bytecode size reduction is calculated.

4) Finally, the combination of patterns that gives the highest bytecode size reduction is selected and used for the generation of the compressed code.

\section{A. Non-Parameterized Pattern Discovery}

The non-parameterized pattern discovery can be reduced into a simple string search problem. Specifically, consider a finite set $\Sigma=\left\{c_{1}, \ldots, c_{\Omega}\right\}$ consisting of $\Omega=|\Sigma|$ individual characters. An arbitrary string over the set $\Sigma$ is any sequence $S_{j}=\left\{s_{j k}\right\}_{k=1}^{L_{j}}$ of length $L_{j}$, where $s_{j k} \in \Sigma$ denotes the character at the $k$-th position of the sequence $S_{j}$. Let $\mathcal{S}=$ $\left\{S_{1}, \ldots, S_{M}\right\}$ be a set of $M$ sequences of length $L_{1}, \ldots, L_{M}$, respectively. In our case, $\mathcal{S}$ denotes the set of basic blocks of the Java bytecode, identified during the first step of the code compression process. Then, the pattern discovery amounts to finding common substrings that are repeated in the sequences of $\mathcal{S}$. Suppose that we search for substrings of a variable length $k=2, \ldots, K$. Then, to locate them we perform the following tasks:

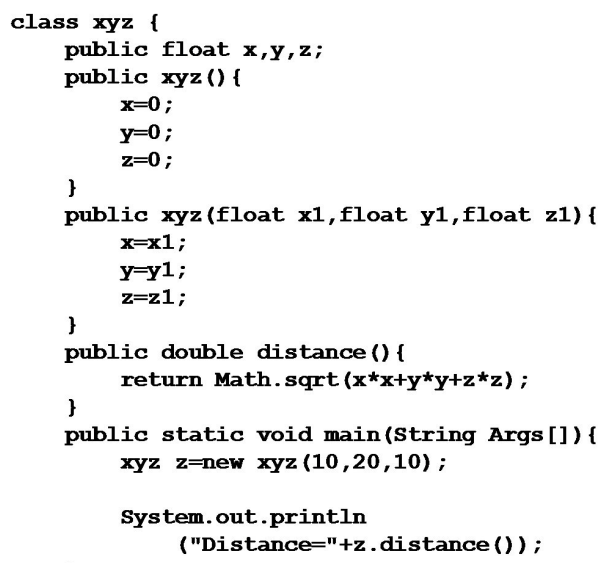

(a) Source code

Compiled from "xyz.java"

class xyz extends java.lang.object

public double distance();

Code:

1: aload 0

4: getfield 02

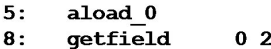

9: fmul

10: aload 0

: getfield 0

14: aload 0

17: getfiēeld 03

18: fmul

19: fadd

20: aload 0

23: getfield

24: aload_0

27: getfield 04

29: fadd

30: f2d

33: invokestatic 0

34: dreturn

(b) Compiled bytecode for the distance method.

Fig. 1. A simple Java program.

1) For each $k=2, \ldots, K$, obtain the collection $X_{k}=$ $\left\{x_{i}\right\}_{i=1}^{n_{k}}$ of $k$-length substrings of the sequences that belong in $\mathcal{S}$, by sliding a window of size $k$ in each sequence $S_{j} \in \mathcal{S}$. For every $S_{j}$, the resulted number of substrings is $L_{j}-k+1$. Hence, the cardinality of $X_{k}$ is $n_{k}=\left|X_{k}\right|=\sum_{j=1}^{M}\left\{L_{j}-k+1\right\}$.

2) Then, the set of non-parameterized patterns $P$ is calculated by searching within each collection $X_{k}$ for identical substrings. The union of the identical substrings obtained during this step constitutes $P$.

To accomplish the first of the above tasks we have to perform $\sum_{k=2}^{K} \sum_{j=1}^{M}\left\{L_{j}-k+1\right\}=\sum_{k=2}^{K}\left\{\left|X_{k}\right|\right\}$ sliding steps. Moreover, to accomplish the second task, we have to perform an overall of $\sum_{k=2}^{K} \sum_{i=0}^{\left|X_{k}\right|-1}\left\{\left|X_{k}\right|-i-1\right\}=\sum_{k=2}^{K}\left\{\left|X_{k}\right| \times\right.$ $\left.\left(\left|X_{k}\right|-1\right) / 2\right\}$ substring comparisons.

All the possible combinations of the patterns retrieved are $2^{|P|}$. The subset of $P$ that gives us the highest bytecode size reduction is stored in the dictionary. The dictionary we use is actually a table of characters. Each table element is used to hold a pattern instruction opcode or operand. Moreover, there are table elements that contain a special character used to signify the end of a pattern; hereafter, we use the term END_OF_PATTERN to refer to that character. The occurrences 
of each pattern are substituted in the original code by a character (i.e., one byte) that indexes the dictionary element that contains the first byte of the pattern. For the selection of the indexing characters we employ the typical approach of Clausen et al., which amounts to using unused bytecode instruction opcodes [4]. Different standard Java platforms for embedded systems comprise unused instruction opcodes, whose number ranges from 52 to 152 . As discussed by Clausen et al., the number of unused opcodes limits the number of patterns that can be used. However, this problem can be alleviated by using a second character, along with the ones that correspond to unused instruction opcodes.

Getting to our example scenario, the bytecode for the distance method (Figure 1(b)) constitutes a basic block as it does not comprise any branch operations. Alphabet $\Sigma$ consists of 12 characters that encode the various opcodes (e.g., get field, aload_0, etc.) and operands (e.g., 0, 2, 3, 4, 5) used in the basic block. The basic block contains patterns of length 4 . Since the overall size of the basic block is 34 characters (i.e., 34 bytes), the set of substrings of length 4 for this basic block contains 31 elements. The set of patterns that results from the 31 substrings includes the 3 patterns given in Figure 2(a).

Let us consider the first of the three patterns. When stored in the dictionary the required space is 4 bytes, plus one more byte for the END_OF_PATTERN character. Since this pattern appears twice in the examined Java bytecode, two bytes are needed in the compressed bytecode to index into the dictionary the position of the pattern. Hence, the gain from substituting the occurrences of the first pattern in the original bytecode is 2 occurences $\times 4$ bytes $-(5$ dictionary bytes + 2 indexing bytes $)=1$ byte. Thus, compressing the bytecode with respect to the first pattern saves us 1 byte. If we repeat the same procedure for the other two patterns, we can save two more bytes and the final bytecode will be 31 bytes. Therefore, the bytecode size reduction obtained is $8.82 \%$. As we demonstrate in the following subsection, this is much smaller compared to the reduction obtained by using the parameterized pattern discovery technique.

\section{B. Parameterized Pattern Discovery}

The parameterized pattern discovery technique is actually an extension of the non-parameterized one. It starts from the point where we have already identified the different collections $X_{2}, \ldots X_{K}$ of substrings of the sequences that belong in $\mathcal{S}$. Following, the discovery of parameterized patterns can be viewed as a clustering problem in the sense of searching for disjoint subsets (clusters) in each set $X_{k}$ that are characterized by a high degree of similarity among the samples that they enclose. Several algorithms have been proposed for clustering discrete data [24], [5], [6]. Among them, we selected the agglomerative clustering (AC) [5], [6], which is a hierarchical approach that relies on the bottom-up generation of a tree-like structure of clusters.

AC is performed for every $X_{k}$ towards obtaining a subset of candidate patterns $P_{k}$. Specifically, AC starts with a set of $\left|X_{k}\right|$ clusters (leaf nodes), each one containing one bytecode substring $x_{i}$ from the set $X_{k}$. Following, a multinomial distribution with $\theta_{v}$ parameters is generated for each cluster $v$. In particular, $\theta_{v}$ can be seen as a two-dimensional matrix of size $k \times|\Omega|$. The rows of the matrix correspond to the $k$ elements of the samples of $v$ (i.e., $x_{i}$, in the first step of the algorithm), while the columns correspond to the different characters of $\Omega$ that can become the values of each element. Then, the value of a matrix element $\theta_{v}[m, l]$ denotes the probability of observing character $c_{l}$ at position $m$ of the samples of $v$. Specifically, $\theta_{v}[\mathrm{~m}, \mathrm{l}]$ is maximum-likelihood (ML) estimated as follows:

$$
\theta_{v}[m, l]=n_{v m l} / n_{v}
$$

$n_{v m l}$ counts the number of occurrences of the character $c_{l} \in \Omega$ at the $m$-th position of the $n_{v}$ samples of cluster $v$. Formally:

$$
n_{v m l}=\sum_{x_{i} \in v} \delta_{i m l}, \text { where } \delta_{i m l}= \begin{cases}1 & \text { if } x_{i m}=c_{l} \\ 0 & \text { otherwise }\end{cases}
$$

In the first step of $\mathrm{AC}, n_{v}=1$ and each row $m$ of the $\theta_{v}$ matrix contains one element equal to 1 . The values of all the other elements of the $m$-th row are 0 . Taking our example program of Figure 1, suppose that we search for patterns, whose maximum length is 9 bytes. AC must be applied in the different collections $X_{2}, \ldots, X_{9}$ that contain bytecode substrings of length $2, \ldots, 9$, respectively. For the case of $X_{9}$, AC results in creating leaf clusters, each one of which includes a sequence of length 9 . Some of these clusters are given in Figure 2(b) (note that in order to simplify the figure we used A, G and F to denote the opcodes, aload_0, getfield and fmul, respectively). Let $v$ be the first leaf cluster from the left side of the figure. Then, $\theta_{v}$ is a $9 \times 12$ matrix. Similar matrices are created for the rest of the leaf clusters of Figure 2(b).

At each next step of AC, the algorithm searches the current set of clusters to identify the two most similar ones $v, u$ that can be merged into a new cluster denoted by $v \cup u$. In our example, the substrings of the first and the third cluster differ only in the operands that reside in their 4-th and 8-th positions. These clusters can be merged into a new cluster that contains the aforementioned substrings. Moreover, the 4th and 8-th rows of the $\theta_{v \cup u}$ matrix comprise two non-zero valued elements. Consequently, the $\theta_{v \cup U}$ matrix represents a parameterized pattern of the two substrings, which contains two wildcards in place of its 4 -th and 8-th elements. A similar merge takes place in the third step of the algorithm. Let $w$ be the 4-th leaf cluster from the left of Figure 2(b). This cluster is merged with the one created in the previous step into a new one that is still represented by the parameterized pattern created in the previous step.

The distance between two clusters is formally defined as follows [5], [6]:

$$
D(v, u)=L_{v}\left(\theta_{v}\right)+L_{u}\left(\theta_{u}\right)-L_{v \cup u}\left(\theta_{v \cup u}\right)
$$

The quantity $L_{v}\left(\theta_{v}\right)$ represents the log-likelihood value that characterizes the cluster $v$ and is given by the following formula:

$$
L_{v}\left(\theta_{v}\right)=\sum_{x_{i} \in v} \sum_{m=1}^{k} \sum_{l=1}^{|\Omega|} \delta_{i m l} \log \theta_{v}[m, l]
$$




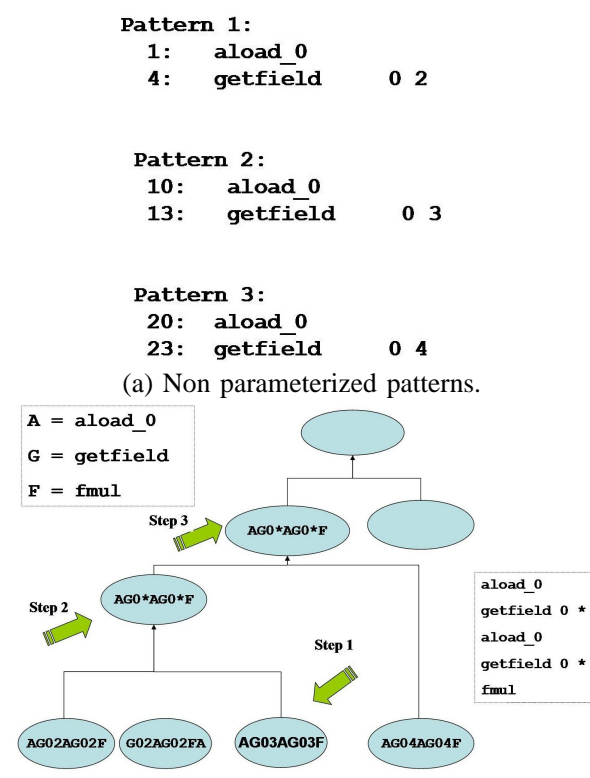

(b) Parameterized patterns.

Fig. 2. Patterns discovered for the distance method.

In general, a wildcard in the $i$-th position of a parameterized pattern that characterizes a cluster signifies that the substrings of the cluster differ in their $i$-th byte. The pattern may contain more than one consecutive wildcards, if the substrings of the cluster differ in more than one consecutive bytes. The AC algorithm may further construct non-parameterized patterns by merging clusters that contain identical substrings.

The algorithm terminates when no pair of nodes is allowed to be further merged. To assess the final set of patterns and obtain $P_{k}$ we perform a depth-first visit upon the nodes of the constructed tree. In particular, starting from the high level nodes, we traverse each subtree until finding the first cluster (node) $v$, whose multinomial density parameters $\theta^{v}$ represent a required degree of similarity. This is done by setting a threshold $T(k)$ to the number of non-wildcard elements of the pattern that is represented by the node that we look for. The value for $T(k)$ should be experimentally determined ${ }^{1}$. Once such a node is found, it is stored in $P_{k}$. By construction, $P_{k}$ may comprise patterns that overlap. More precisely, two patterns in $P_{k}$ may refer to the same opcode or operand of the original bytecode. Overlapping patterns may contribute differently in the overall bytecode size reduction and therefore they are included in $P_{k}$. At the end of $\mathrm{AC}$, the retrieved patterns are further simplified. Specifically, if $P_{k}$ contains a pattern with a wildcard in place of the first (or the last) byte, this pattern is substituted with the pattern that results from removing this wildcard. Therefore, the resulting set $P_{k}$ contains patterns that do not start or end with a wildcard. Nonparameterized patterns may also result from the simplification procedure when it is applied in patterns that contain wildcards only in place of their first or last bytes.

The $P_{2}, \ldots, P_{K}$ subsets of significant patterns obtained

${ }^{1}$ In our particular experiments discussed in Section IV, a value of $T(k)=$ $\lceil k / 2\rceil$ was sufficient for obtaining good percentages of bytecode size reduction. from the application of $\mathrm{AC}$ to the $X_{2}, \ldots, X_{K}$ collections of substrings are finally merged into a single set of significant patterns $P$. Again, $P$ may comprise overlapping patterns. Moreover, $P$ may comprise nested patterns, in the sense that a pattern $p$ that was originally included in $P_{i}$ exactly matches with a part of a pattern $q$ that was originally included in $P_{j}$ where $j>i$. The elements of $P$ are combined during the last two steps of the code compression process mentioned at the beginning of this section.

As in the case of the non-parameterized patterns technique, the total number of possible combinations of patterns is $2^{|P|}$. The subset of them that gives us the highest bytecode size reduction is stored in the dictionary, whose structure is however slightly different compared to the one used in the non-parameterized pattern discovery technique. Specifically, the dictionary is still a table of characters. Each table element holds a pattern instruction opcode or operand and there are elements that contain the END_OF_PATTERN character. The wildcards are not stored in the pattern. Instead of wasting one table element for storing a value that signifies the existence of a wildcard, we only spend 1 bit. Specifically, for patterns of maximum length $K$ we use $\lceil(K-2) / 8\rceil$ table elements at the beginning of each pattern to encode the positions of the wildcards within it. Recall that the patterns produced by AC do not contain wildcards in their first and last bytes; hence, a pattern of maximum length $K$ may contain at most $K-2$ wildcards. Then, the value of the $i$-th bit of a table element that encodes the positions of wildcards within the pattern is 1 , if the pattern contains a wildcard in the $i$-th position. Hence, for patterns of maximum length 10 we use 1 extra table element. Similarly, for patterns whose length is between 11 and 18, we use 2 extra table elements and so on. In the original bytecode the occurrences of a pattern are substituted by a character (i.e., one byte) that indexes the dictionary elements that encode the positions of wildcards within the patterns. The indexing byte is followed by the actual values of these wildcards. As indexing characters we use unused bytecode instruction opcodes [4].

Summarizing, the most important concept of AC is the intercluster distance $D(u, v)$, which we use as a basic criterion for merging clusters during the construction of the tree. The distance used (Eq. 3) is derived from a probabilistic model; it reflects the likelihood decrease that results by merging the clusters $u$ and $v$. During each one of its steps, the algorithm merges the best pair of clusters, i.e., those having the lower likelihood decrease when putting together. AC has several advantages as it requires $O\left(\left|X_{k}\right|\right)$ memory for every $X_{k}, k=$ $2, \ldots, K$, while its complexity is typically quadratic to $\left|X_{k}\right|$ [5]. Nevertheless, the application of other statistical methods for finding patterns in Java bytecode constitutes one of our future directions in this subject area.

Returning to our example scenario suppose that we use the pattern identified in Figure 2(b) to compress our simple Java program. The pattern appears in three bytecode sequences in the program. Originally, for these sequences we need $3 \times 9$ bytes $=27$ bytes. In the dictionary we need 7 bytes to store the non-wildcard pattern elements and one extra byte to encode the positions of the wildcards.

Finally, we need 1 byte for the END_OF_PATTERN character. 


\begin{tabular}{|c|c|c|c|c|c|c|c|c|}
\hline Java Program & $\begin{array}{l}\text { Binary } \\
\text { Search }\end{array}$ & $\begin{array}{l}\text { Bub } \\
\text { Sort }\end{array}$ & ble & $\begin{array}{l}\text { Fibonacei } \\
\text { Numbers }\end{array}$ & $\begin{array}{l}\text { Minimu } \\
\text { m in } \\
\text { Array }\end{array}$ & $\begin{array}{l}\text { Matrix } \\
\text { Addition }\end{array}$ & $\begin{array}{l}\text { Prime } \\
\text { Numbers }\end{array}$ & $\begin{array}{l}\text { Selection } \\
\text { Sort }\end{array}$ \\
\hline $\begin{array}{l}\text { class file size } \\
\text { (bytes) }\end{array}$ & 789 & 871 & & 712 & 758 & 840 & 576 & 889 \\
\hline $\begin{array}{l}\text { bytecode size } \\
\text { (bytes) }\end{array}$ & 56 & 60 & & 27 & 30 & 29 & 27 & 71 \\
\hline \multicolumn{9}{|c|}{ (a) Class file and bytecode sizes. } \\
\hline Java Program & $\begin{array}{l}\text { Binar } \\
\text { Searc }\end{array}$ & & $\begin{array}{l}\text { Bubble } \\
\text { Sort } \\
\end{array}$ & $\begin{array}{ll}\text { Fibonacei } \\
\text { Numbers }\end{array}$ & $\begin{array}{l}\text { Minimum } \\
\text { in Array }\end{array}$ & $\begin{array}{ll}\text { n } & \begin{array}{l}\text { Matrix } \\
\text { Addition }\end{array} \\
\end{array}$ & $\begin{array}{ll}\text { Prime } \\
\text { Numbers } \\
\end{array}$ & $\begin{array}{l}\text { Selection } \\
\text { Sort } \\
\end{array}$ \\
\hline parameterized & 14.28 & & $6.66 \%$ & $3.70 \%$ & $3.33 \%$ & $8.82 \%$ & $4.83 \%$ & $7.04 \%$ \\
\hline $\begin{array}{l}\text { non- } \\
\text { parameterized }\end{array}$ & 10.71 & & $6.66 \%$ & $0.00 \%$ & $0.00 \%$ & $1.47 \%$ & $0.00 \%$ & $0.00 \%$ \\
\hline
\end{tabular}

(b) Percentage of bytecode size reduction.

TABLE I

USING THE NON-PARAMETERIZED AND THE PARAMETERIZED TECHNIQUES TO COMPRESS A TYPICAL SET OF ALGORITHMS IMPLEMENTED IN JAVA.

In the bytecode, each pattern occurrence is replaced by three bytes, one for indexing the directory and two for the actual values of the two wildcards. Thus, in the compressed code we need 9 bytes for specifying the positions of the pattern occurrences in the bytecode and 9 bytes for storing the pattern in the dictionary. This means that the compression results in saving 9 bytes. Hence, the use of parameterized patterns gives us a much better bytecode size reduction (26.47\%), compared to the one $(8.82 \%)$ obtained in the case of the nonparameterized pattern discovery technique.

To further motivate the investigation of the advanced parameterized pattern discovery technique we applied it in a set of simple Java programs that realize standard algorithms. The sizes of these simple programs are given in Table I(a). The percentages of the bytecode size reduction we obtained in these examples are given in Table I(b). It should be noted that these results are optimal in the sense that we performed all possible combinations of the retrieved patterns towards locating the subset of these patterns that gives the highest bytecode size reduction. Once more, we can observe that the parameterized pattern discovery technique results in better percentages of bytecode size reduction, compared to the ones obtained from the non-parameterized one. It is worth noticing that in some of the examples (e.g., fibonacci numbers, selection sort, etc.), the non-parameterized technique completely failed to locate any patterns, while the parameterized technique results in a respectable percentage of bytecode size reduction.

\section{Bytecode decompression}

The bytecode decompression procedures for the techniques we discussed in this section are quite straightforward. In the case of the non-parameterized technique, the decompression module is initialized with a given dictionary. The module provides an operation that accepts as input an index to the dictionary. This particular operation should be called by the main loop of the Java bytecode interpreter, upon the discovery of a byte that corresponds to an unused bytecode opcode. The decompression module uses the input index to find the first byte of the pattern. This corresponds to the first instruction opcode contained in the pattern. The decompression module reasons based on the given opcode about the number of bytes that need to be fetched from the dictionary. Following, the required number of bytes are fetched from the dictionary towards forming an instruction. The remaining instructions that constitute the particular pattern are decompressed similarly. The overall decompression procedure ends up by fetching the END_OF_PATTERN element from the dictionary.

For the parameterized technique, the decompression is similar. The decompression module is also initialized with a given dictionary and the maximum length used for deriving the patterns contained in this dictionary. The decompression module accepts as input an index to the dictionary. Following, it uses the input index to repeatedly fetch from the dictionary a number of elements that encode the positions of the wildcards within the indexed pattern. The number of these elements depends on the maximum length used for discovering the patterns contained in the dictionary (by default, the maximum length of patterns is 9 and therefore one byte is fetched). The byte that follows the elements that encode the positions of wildcards is the first instruction opcode contained in the pattern. As previously, the decompression module figures out about the number of bytes required for forming the first instruction that should be executed. Some of the required bytes are fetched from the dictionary, while some others are found in the compressed code. Specifically, the $i$-th byte is fetched from the dictionary if the value of the $i$-th bit of the bytes that encode the positions of wildcards within the pattern is 0 . Otherwise, the $i$-th byte is found in the compressed code. The remaining instructions that constitute the particular pattern are decompressed similarly. Again, the overall decompression procedure ends up by fetching the END_OF_PATTERN element from the dictionary.

\section{Assessment}

Although some benefits from using the parameterized pattern discovery technique are evident from the simple examples discussed in Section III, we further conducted experiments involving a real-world case study. Specifically, the target of our investigation is the MIDP (Mobile Information Device Profile) v2.0 reference implementation from Sun Microsystems ${ }^{2}$. MIDP is part of the Java 2 Platform Micro Edition (J2ME) and relies on CLDC (Connected Limited Device Configuration). It provides a basic environment for the development of Java applications for mobile information devices (MIDs) such as mobile phones and PDAs. The MIDP reference implementation consists of 11 packages. In this section, we present the results we obtained from 6 of these packages, which we consider as the core of MIDP. The basic characteristics of each package (size of class file and bytecode size) are given in Table II. The sizes of the particular Java class files are representative, considering real world applications aimed at mobile and embedded devices. Specifically, the standard JTWI (Java Technology for the Wireless Industry ${ }^{3}$ ) specification sets the limit of a standard-size-application to 64Kbytes. JTWI actually defines a standard framework for the development of mobile applications and MIDP is part of it. Applications that are

\footnotetext{
${ }^{2}$ http://java.sun.com/products/midp/

${ }^{3}$ http://java.sun.com/products/jtwi/
} 


\begin{tabular}{lcc}
\hline \multicolumn{1}{c}{ MIDP Package } & $\begin{array}{c}\text { Class } \\
\text { file size } \\
\text { (bytes) }\end{array}$ & $\begin{array}{c}\text { Bytecode } \\
\text { size } \\
\text { (bytes) }\end{array}$ \\
\hline java.io & 19568 & 3454 \\
\hline java.lang & 27714 & 3998 \\
\hline javax.microedition.io & 4599 & 509 \\
\hline javax.microedition.media & 2065 & 362 \\
\hline javax.microedition.midlet & 2025 & 121 \\
\hline javax.microedition.pki & 2563 & 184 \\
\hline
\end{tabular}

TABLE II

MIDP PACKAGES BASIC FEATURES.

under the limit of 64Kbytes are guaranteed to work correctly over any kind of device that complies with JTWI. Regarding the MIDP packages, java.io provides classes for input and output through data streams. The java.lang package consists of basic language classes coming from J2SE (Java 2 Standard Edition), javax.microedition.io includes networking support relying on CLDC. The javax.microedition.media package allows accessing device-dependent resources for multimedia processing. The javax.microedition.midlet package defines the basic MIDP application model. Finally, javax.microedition.pki enables managing certificates, used for securing connections.

The goal of our experiments was twofold:

1) To investigate the benefits and the cost of the parameterized pattern discovery technique, measured in terms of the bytecode size reduction obtained and the time spent for discovering and combining patterns, respectively.

2) To study the cost of the decompression procedure that comes along with the parameterized technique in terms of the time overhead, introduced in the execution of Java bytecode.

\section{A. Bytecode Compression}

To evaluate the benefits and the cost of the parameterized pattern discovery technique we performed two different sets of experiments. The first one aims at evaluating the impact of using patterns that contain a variable number of wildcards in the compression of MIDP. The second set of experiments aims at investigating the impact of the increasing length of the patterns in the compression of MIDP. In the first set of experiments, the maximum length of the patterns is set to 9 , while in the second one we increase this length from 9 to 11 . In both sets, the threshold for non-wildcard elements in the patterns was set to $T(k)=\lceil k / 2\rceil$.

The strong point of the advanced parameterized pattern discovery technique is its ability to track-down a rich variety of patterns, whose combination may lead to more effective bytecode size reduction. This fact is particularly highlighted in Tables III(a) and (b), which give the total number of patterns and the number of non-parameterized patterns discovered in the MIDP packages. Table III(a) specifically refers to the first set of experiments where the maximum length of the patterns was 9 , while Table III(b) refers to the second set of

\begin{tabular}{|c|c|c|c|}
\hline \multirow{2}{*}{ MIDP Package } & \multicolumn{2}{|c|}{$\begin{array}{c}\text { Number of patterns found } \\
\text { (pattern length }=2 \text { to 9) }\end{array}$} & \multirow{2}{*}{$\begin{array}{l}\text { Execution time } \\
\text { for AC (sec) }\end{array}$} \\
\hline & parameterized & $\begin{array}{c}\text { non- } \\
\text { parameterized }\end{array}$ & \\
\hline java.io & 4408 & 1870 & 6903 \\
\hline java.lang & 4160 & 2322 & 25403 \\
\hline javax.microedition.io & 66 & 41 & 632 \\
\hline javax.microedition.media & 541 & 245 & 500 \\
\hline javax.microedition.midlet & 531 & 295 & 12 \\
\hline javax.microedition.pki & 91 & 23 & 55 \\
\hline \multicolumn{4}{|c|}{ (a) Maximum length of patterns $=9$. } \\
\hline \multirow{2}{*}{ MIIDP Package } & \multicolumn{2}{|c|}{$\begin{array}{l}\text { Number of patterns found } \\
\text { (pattern length = 2 to 11) }\end{array}$} & \multirow{2}{*}{$\begin{array}{l}\text { Execution time } \\
\text { for AC (sec) }\end{array}$} \\
\hline & parameterized & $\begin{array}{c}\text { non- } \\
\text { parameterized }\end{array}$ & \\
\hline java.io & 4583 & 1981 & 8701 \\
\hline java.lang & 4375 & 2504 & 32639 \\
\hline javax.microedition.io & 99 & 43 & 818 \\
\hline javax.microedition.media & 577 & 253 & 646 \\
\hline javax.microedition.midlet & 533 & 295 & 15 \\
\hline javax.microedition.pki & 98 & 23 & 74 \\
\hline
\end{tabular}

(b) Maximum length of patterns $=11$.

TABLE III

COMPARING THE NUMBERS OF PARAMETERIZED AND NON-PARAMETERIZED PATTERNS DISCOVERED IN MIDP.

experiments where the maximum length of the patterns was 11. In general, we can observe that increasing the maximum length of the patterns results in increasing the overall number of patterns found.

Nevertheless, the time required by the advanced parameterized pattern discovery technique for the discovery of patterns is quite high (Tables III(a) and (b)). Moreover, the discovery of a rich variety of patterns also increases the complexity of combining these patterns to obtain a good bytecode size reduction. Therefore, an issue that should be studied in our assessment is whether the discovered patterns are useful, worthy of the time required for discovering and combining them.

As already discussed, the complexity for finding all possible combinations of patterns is $2^{|P|}$. In the first set of experiments, the average number of parameterized patterns found per MIDP class is 157 . On the other hand, the average number of nonparameterized patterns found per MIDP class is 85.6. If we assume that each combination of patterns requires 1 usec to be performed, then the assessment of all possible combinations of parameterized patterns would require $5.39864 * 10^{31}$ hours to complete. Similarly, the assessment of all combinations of non-parameterized patterns would require $1.69879 * 10^{10}$ hours. The assumption that the assessment of a particular combination of patterns requires $1 \mathrm{usec}$ is rather optimistic and refers to combinations of 2 patterns. As we can observe in the experiments detailed later the required time depends on the number of combined patterns and it is usually higher than 1 usec. Therefore, for both the parameterized and the nonparameterized sets of patterns found in MIDP, the assessment of all possible combinations is virtually impossible. To deal with this particular problem we investigate the use of two heuristics. The purpose of the heuristics is to allow us to 
efficiently obtain good suboptimal bytecode size reduction.

In both heuristics, we sort an overall set of patterns $P$ based on the bytecode size reduction provided by these patterns when they are used in isolation for the compression of the bytecode within which they were found. In a sense, we quantitatively group/cluster the patterns with respect to the bytecode size reduction that they provide. Following, in the first heuristic we start from the pattern that offers the best bytecode size reduction and we examine its combination with the secondbest pattern. If the bytecode size reduction obtained using the two patterns is greater than the one obtained from the first pattern we keep the second pattern as well. Otherwise, the second-best pattern is useless. We follow the same procedure for the rest of the sorted patterns, ending up with a set of patterns that gives us a suboptimal bytecode size reduction. The second heuristic amounts to performing the first heuristic $|P|$ times. During each iteration $i: i=1, \ldots|P|$ we start from the $i$-th best of the sorted patterns and we proceed by checking the usefulness of combining it with the $i+1, i+$ $2, \ldots,|P|, 1,2, \ldots, i-1$ patterns. At the end of this procedure we end up with $|P|$ sets of patterns, amongst which we select the one that gives us the highest bytecode size reduction.

In general, the impact of the second heuristic in the overall time required for the bytecode compression is expected to be much higher than the impact of the first heuristic (since the application of the second heuristic consists of applying the first heuristic $|P|$ times). On the other hand, the combinations of patterns examined by the second heuristic are a superset of the combinations of patterns examined by the first heuristic. Hence, the percentage of bytecode size reduction obtained from the application of the second heuristic is expected to be at least as good as the percentage of bytecode size reduction obtained from the application of the first heuristic.

1) Experimental results - 1st set of experiments: In the first set of experiments we used as input to both heuristics the full set of patterns, $P_{\text {param }}$, discovered by the parameterized pattern discovery technique in each one of the MIDP packages. Moreover, we used as input to both heuristics the set of non-parameterized patterns, $P_{n o n-p a r a m}$, discovered in each one of the MIDP packages. To compare the contribution of patterns that contain a variable number of wildcards, against the contribution of patterns that contain a fixed number of wildcards we further constructed four input sets, $P_{1 w}, P_{2 w}$, $P_{3 w}$ and $P_{4 w}$, consisting of patterns that contain one, two, three and four wildcards, respectively. $P_{1 w}, P_{2 w}, P_{3 w}$ and $P_{4 w}$ were constructed from the full set of patterns $P_{\text {param }}$ that resulted from AC. Finally, to investigate the impact of the increasing number of wildcards in the compression of the MIDP packages we constructed three more input sets, $P_{1-2 w}$, $P_{1-3 w}$ and $P_{1-4 w}$, consisting of patterns that contain oneto-two, one-to-three and one-to-four wildcards, respectively. $P_{1-2 w}, P_{1-3 w}$ and $P_{1-4 w}$ were also constructed from the full set of patterns $P_{\text {param }}$ that resulted from AC.

Figure 3 compares the results we obtained per MIDP package from the application of the first heuristic in $P_{\text {param }}$, $P_{n o n-p a r a m}, P_{1 w}, P_{2 w}, P_{3 w}$ and $P_{4 w}$ (more detailed results regarding the bytecode size reduction obtained per different class of the MIDP packages are given in Appendix I).
Specifically, the time required to combine the patterns (Figure 3(b)) is acceptable in every case. However, the bytecode size reduction we obtained for each package highly depends on the patterns used. In particular, in some packages (e.g., javax.microedition.midlet), $P_{\text {param }}$ gives the best bytecode size reduction. In other packages (e.g., java.io), the nonparameterized set, $P_{\text {non-param }}$, performs better. Finally, there are also packages where the sets that contain patterns with fixed numbers of wildcards give the best bytecode size reduction (e.g., $P_{1 w}$ and $P_{2 w}$ in javax.microedition.pki). Therefore, the first heuristic is quite fast but rather unpredictable regarding the set of patterns that should be used to obtain the highest bytecode size reduction. Moreover, in most packages the first heuristic appears incapable of taking advantage of the flexibility provided by the large variety of patterns contained in $P_{\text {param }}$.

The results obtained from the application of the second heuristic in $P_{\text {param }}, P_{n o n-p a r a m}, P_{1 w}, P_{2 w}, P_{3 w}$ and $P_{4 w}$ are given in Figure 4 (more details regarding the bytecode size reduction obtained per different MIDP class are given in Appendix I). As expected, the time required to combine patterns (Figure 4(b)) is much higher than the time spent when using the first heuristic. However, it is still reasonable compared to the time required for deriving all possible combinations of patterns. The bytecode size reduction we obtained is generally better than the corresponding reduction obtained from the application of the first heuristic. The use of $P_{\text {param }}$ gives the highest bytecode size reduction in all packages, except for the javax.microedition.pki one (Figure 4(a)). Hence, the second heuristic renders the use of $P_{\text {param }}$ more beneficial. The reduction obtained from the sets that contain patterns with fixed numbers of wildcards is in most packages poorer than the reduction resulted from $P_{\text {param }}$. However, amongst $P_{1 w}$, $P_{2 w}, P_{3 w}$ and $P_{4 w}$, the first two sets give better bytecode size reduction. Hence, patterns with a relatively small number of wildcards contribute more in reducing the size of the MIDP packages.

Figures 5 and 6 compare the results we obtained from the application of the first and the second heuristic in $P_{\text {param }}$, $P_{n o n-p a r a m}, P_{1-2 w}, P_{1-3 w}$ and $P_{1-4 w}$ (more details can be found in Appendix I). Regarding the two heuristics, the observations derived from Figures 3 and 4 are still valid. The bytecode size reduction obtained from the first heuristic is smaller compared to the one obtained from the application of the second heuristic. In both heuristics the reduction obtained in the case of $P_{1-2 w}, P_{1-3 w}$ and $P_{1-4 w}$ is greater than the reduction obtained in the case of $P_{1 w}, P_{2 w}, P_{3 w}$ and $P_{4 w}$. In most cases, $P_{1-2 w}$ gives better bytecode size reduction than $P_{1-3 w}$ and $P_{1-4 w}$. This observation is an additional evidence that patterns with a small number of wildcards result in combinations that give us better bytecode size reduction.

The inclusion of patterns with 3 and 4 wildcards in the set of patterns that contain 1 and 2 wildcards results in worst bytecode size reduction in certain cases because of the overlapping between these patterns. For example, a pattern that contains 3 wildcards could appear in the bytecode in the form of two consecutive patterns that contain 1 and 2 wildcards. In the same bytecode, there may also be individual occurrences 


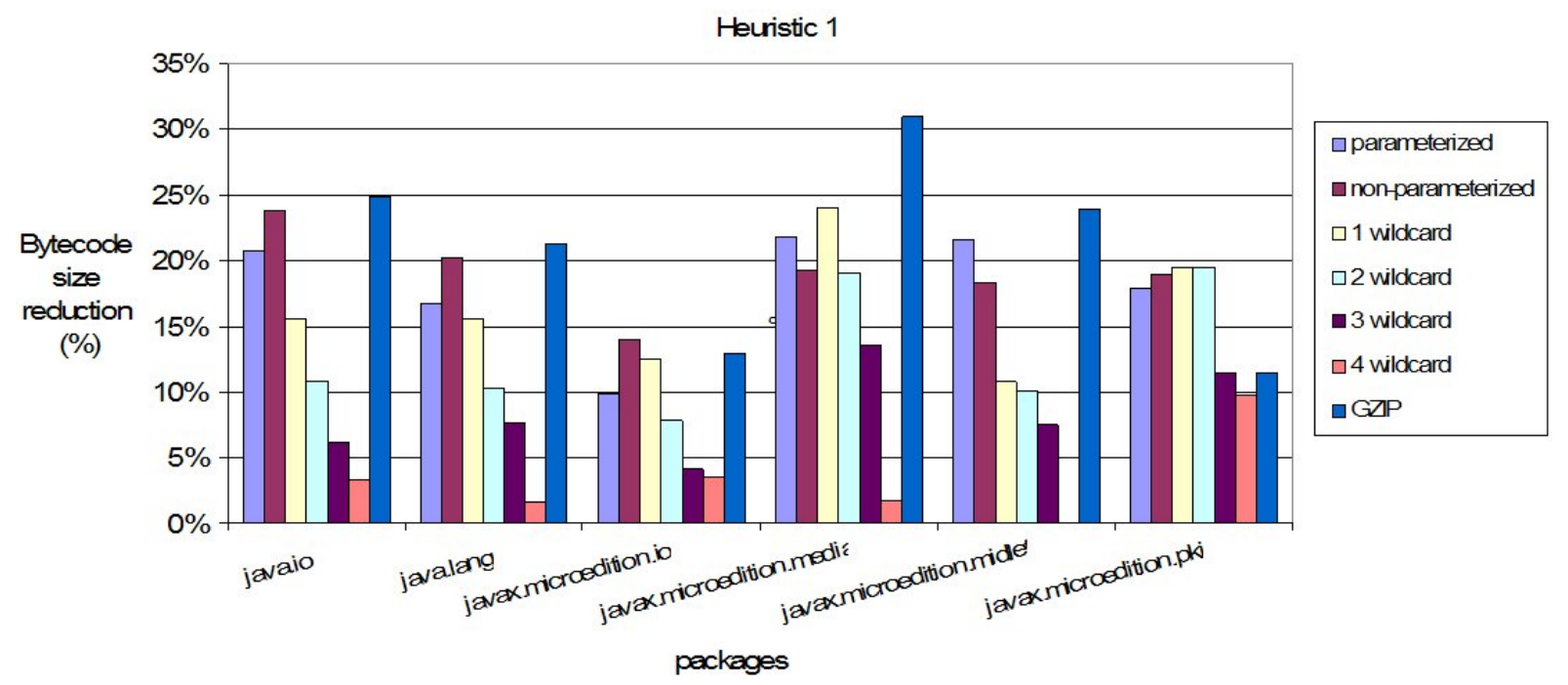

(a) Percentage of bytecode size reduction per MIDP package.

\begin{tabular}{lccccccc}
\hline MIIP Package & Parameterized & $\begin{array}{c}\text { Non- } \\
\text { parameterized }\end{array}$ & $\begin{array}{c}\text { Parameterized } \\
\text { patterns with 1 } \\
\text { wildcard }\end{array}$ & $\begin{array}{c}\text { Parameterized } \\
\text { patterns with 2 } \\
\text { wildcards }\end{array}$ & $\begin{array}{c}\text { Parameterized } \\
\text { patterns with 3 } \\
\text { wildcards }\end{array}$ & $\begin{array}{c}\text { Parameterized } \\
\text { patterns with 4 } \\
\text { wildcards }\end{array}$ \\
\hline java.io & 2.03 & 1.17 & 0.66 & 0.21 & 0.09 & 0.08 \\
\hline java.lang & 3.975 & 7.06 & 1.96 & 0.4 & 0.46 & 0.2 \\
\hline javax.microedition.io & 0.2 & 0.1 & 0.09 & 0.02 & 0.02 & 0.02 \\
\hline javax.microedition.media & 0.27 & 0.18 & 0.05 & 0.01 & 0.01 & 0.01 \\
\hline javax.microedition.midlet & 0.03 & 0.02 & 0.02 & 0.02 & 0.02 & 0.02 \\
\hline javax.microedition.pki & 0.01 & 0.01 & 0.01 & 0.01 & 0.01 & 0.01 \\
\hline
\end{tabular}

(b) Time to combine patterns per MIDP package (sec).

Fig. 3. Experimental results from the application of the first heuristic in $P_{\text {param }}, P_{n o n-p a r a m}, P_{1 w}, P_{2 w}, P_{3 w}$ and $P_{4 w}$.

of the two constituent patterns. If used in isolation ${ }^{4}$, the 2 constituent patterns may result in bytecode size reductions that are smaller than the one that can be obtained from the use of the 3-wildcards-pattern. On the other hand, if both the constituent patterns are used we may get a reduction that is better than the one obtained by the use of the 3-wildcardspattern. However, the selection of the 3-wildcards-pattern may render the selection of the two constituent patterns impossible (e.g. because after the substitution of the 3-wildcards-pattern occurrences, the constituent patterns no longer appear in the bytecode more than once).

Although one would expect that the bytecode size reduction resulted from $P_{1-3 w}$ and $P_{1-4 w}$ would be really close or equal to the one obtained from $P_{\text {param }}$, this is not the case. $P_{1-4 w}$ comprises all the patterns that contain wildcards. However, $P_{\text {param }}$ further comprises the non-parameterized patterns, discovered by AC (Section III B). The contribution of the non-parameterized patterns is quite significant along with the contribution of the patterns that contain 1 and 2 wildcards. This becomes clear with further elaboration on the results obtained from the application of the second heuristic in $P_{\text {param }}$. Specifically, Figure 7 gives the numbers of useful patterns (i.e., the patterns that are finally used for compressing the MIDP packages) that resulted from the second heuristic in relation with their lengths and the number of wildcards that

\footnotetext{
${ }^{4}$ i.e. according to the way that we assess the contribution of patterns in the heuristics
}

they contained.

Figures 3(a), 4(a), 5(a) and 6(a) further compare the results we discussed so far with the ones obtained from the use of GZIP. We used GZIP to compress the bytecode of the different MIDP packages that constitute our case study. The resulted compressed bytecode is not interpretable, in that it can not be executed without being fully decompressed. However, the bytecode size reduction obtained with GZIP is a useful measure towards our assessment. In all figures, we can observe that the percentage of the bytecode size reduction obtained with GZIP is comparable with the one resulted from $P_{\text {param }}$. It is interesting to note that in the case of the first heuristic (Figures 3(a), 5(a)), in most MIDP packages, GZIP performs slightly better than $P_{\text {param }}$ and $P_{\text {non-param }}$. On the other hand, in the case of the second heuristic we see that $P_{\text {param }}$ gives in some MIDP packages (java.io, java.lang packages) better bytecode size reduction than GZIP, while $P_{\text {non-param }}$ gives only at most as good bytecode size reduction as GZIP.

Summarizing the results from the first set of experiments, we can derive the following conclusions. The examined pattern discovery technique allows the discovery of a rich variety of parameterized and non-parameterized patterns. The discovery of the patterns is quite expensive, with respect to the time spent by AC. Moreover, the complexity of selecting and combining patterns is also high. Our experimental results showed that in the case of MIDP, the most useful patterns out of $P_{\text {param }}$ are the non-parameterized ones and the ones that contain 1 


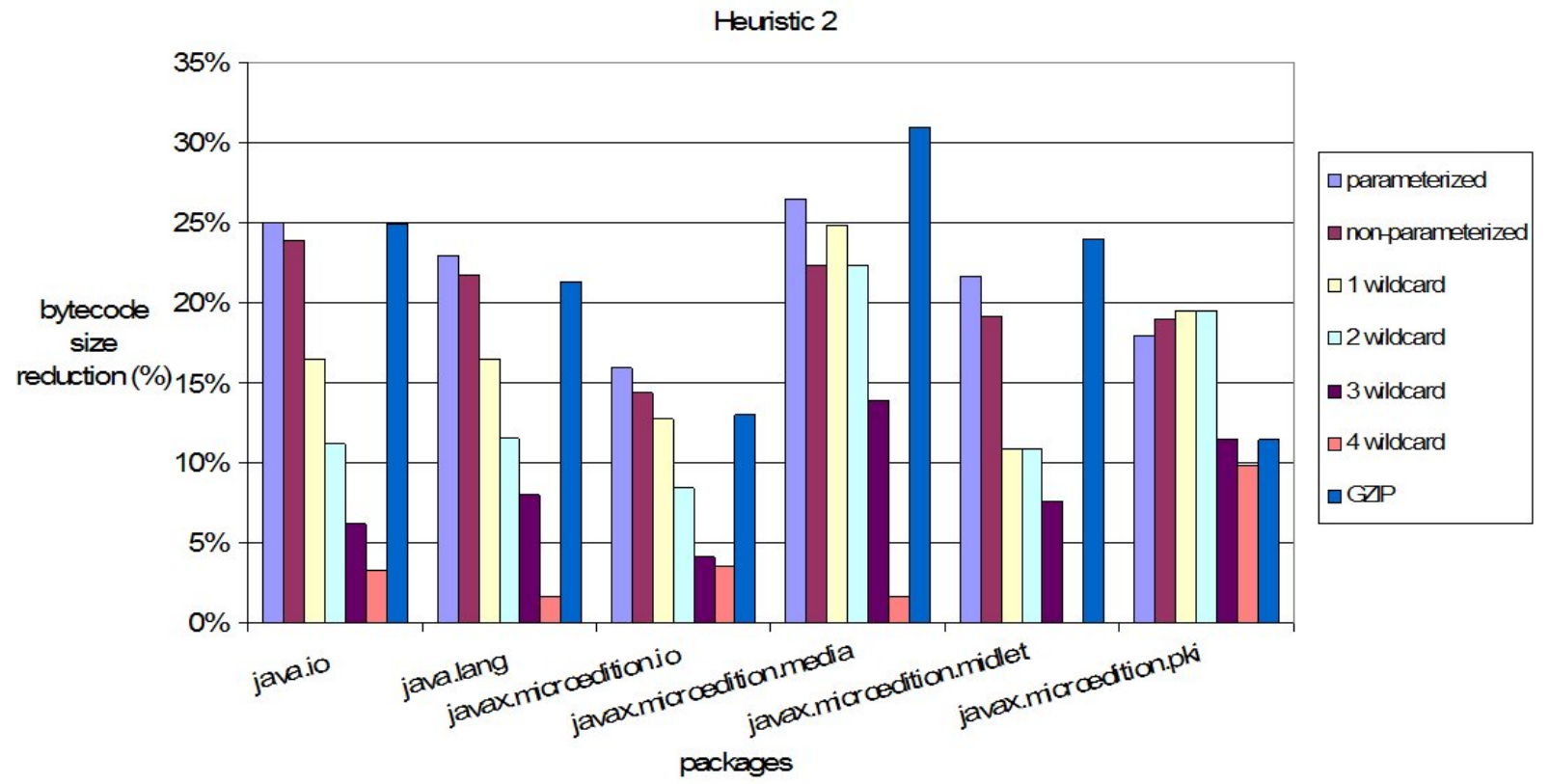

(a) Percentage of bytecode size reduction per MIDP package.

\begin{tabular}{lcccccc}
\hline MIIP Package & Parameterized & $\begin{array}{c}\text { Non- } \\
\text { parameterized }\end{array}$ & $\begin{array}{c}\text { Parameterized } \\
\text { patterns with 1 } \\
\text { wildcard }\end{array}$ & $\begin{array}{c}\text { Parameterized } \\
\text { patterns with 2 } \\
\text { wildcards }\end{array}$ & $\begin{array}{c}\text { Parameterized } \\
\text { patterns with 3 } \\
\text { wildcards }\end{array}$ & $\begin{array}{c}\text { Parameterized } \\
\text { patterns with } \\
\text { 4 widcards }\end{array}$ \\
\hline java.io & 2126.65 & 487.64 & 131.21 & 11.73 & 3.64 & 0.18 \\
\hline java.lang & 12731.71 & 4122.76 & 139.11 & 39.34 & 16.84 & 1.19 \\
\hline javax.microedition.io & 119.94 & 22.24 & 18.47 & 1.05 & 0.07 & 0.07 \\
\hline javax.microedition.media & 0.27 & 0.18 & 0.05 & 0.01 & 0.01 & 0.02 \\
\hline javax.microedition.midlet & 0.12 & 0.02 & 0.02 & 0.02 & 0.02 \\
\hline javax.microedition.pki & 0.01 & 0.01 & 0.01 & 0.01 & 0.01 & 0.01 \\
\hline
\end{tabular}

(b) Time to combine patterns per MIDP package (sec).

Fig. 4. Experimental results from the application of the second heuristic in $P_{\text {param }}, P_{n o n-p a r a m}, P_{1 w}, P_{2 w}, P_{3 w}$ and $P_{4 w}$.

and 2 wildcards. However, this may not be the case in other Java applications, where patterns with more than 2 wildcards may also prove useful. Based on these remarks, a good strategy for balancing the tradeoff between the time spent for discovering/combining patterns and the resulted bytecode size reduction is to apply the technique in an incremental manner. For a given bytecode, instead of using AC for constructing a large set of patterns, containing many wildcards, AC can be customized to construct a smaller set of patterns, containing few wildcards. Starting from this smaller set of patterns, the technique may be used at a later time, if necessary, to discover patterns that contain more wildcards, towards further improving the bytecode size reduction.

2) Experimental results - 2nd set of experiments: In the second set of experiments we used as input to both heuristics the set of patterns of maximum length $11, P_{\text {param }}$, discovered in each one of the MIDP packages. As discussed at the beginning of this subsection, increasing the maximum length of the patterns results in the discovery of more patterns. Moreover, increasing the maximum length implies increasing the time required for discovering the patterns and the time required for combining them. The expected benefit against the time increment is the increment of the bytecode size reduction. However, the previous may not hold; increasing the maximum length of the patterns may result only in a small increment of the bytecode size reduction. In the worst case, it may result in a decrement of the bytecode size reduction. As detailed in Section III B, to encode the positions of wildcards in patterns of maximum length $K$, we have to use $\lceil(K-2) / 8\rceil$ bytes. Therefore, for patterns of maximum length 9,1 byte is needed. On the other hand, for patterns of maximum length 11,2 bytes have to be used. The extra bytes used for encoding the positions of wildcards when the maximum length of the patterns is long may reduce the benefits obtained from using these patterns.

More specifically, Figures 8(a) and (b) give the results we obtained in the case of the MIDP packages. Figure 8(a) refers to the first heuristic, while Figure 8(b) refers to the second heuristic. In both figures, the left axis corresponds to the percentage of the bytecode size reduction increment or decrement resulted from increasing the maximum length of the patterns from 9 to 11 (columns that are under $0 \%$ correspond to bytecode size reduction decrement, while columns that are over $0 \%$ correspond to bytecode size reduction increment; the smaller the columns that are under $0 \%$, the larger the decrement of the bytecode size reduction). The right axis shows the increment of the time required for discovering and combining the patterns when their maximum length is increased from 9 to 


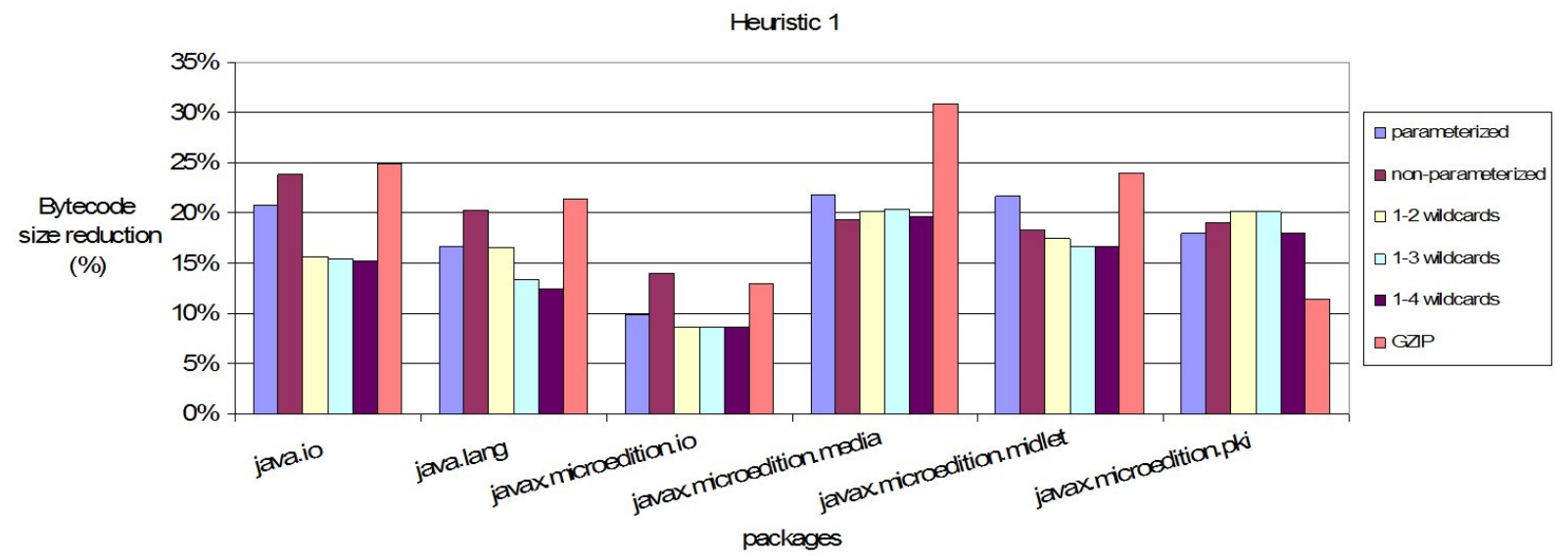

(a) Percentage of bytecode size reduction per MIDP package.

\begin{tabular}{lccccc}
\hline \multicolumn{1}{c}{ MIIDP Package } & Parameterized & $\begin{array}{c}\text { Non- } \\
\text { parameterized }\end{array}$ & $\begin{array}{c}\text { Parameterized } \\
\text { patterns with 1- } \\
\text { 2 wilcards }\end{array}$ & $\begin{array}{c}\text { Parameterized } \\
\text { patterns with } \\
\text { 1-3 widcards }\end{array}$ & $\begin{array}{c}\text { Parameterized } \\
\text { patterns with 1-4 } \\
\text { wildcards }\end{array}$ \\
\hline java.io & 2.03 & 1.17 & 0.84 & 0.91 & 1.02 \\
\hline java.lang & 3.975 & 7.06 & 1.25 & 1.34 & 1.53 \\
\hline javax.microedition.io & 0.2 & 0.1 & 0.11 & 0.12 & 0.14 \\
\hline javax.microedition.media & 0.27 & 0.18 & 0.1 & 0.1 & 0.11 \\
\hline javax.microedition.midlet & 0.03 & 0.02 & 0.02 & 0.02 & 0.02 \\
\hline javax.microedition.pki & 0.01 & 0.01 & 0.01 & 0.02 & 0.03 \\
\hline
\end{tabular}

(b) Time to combine patterns per MIDP package (sec).

Fig. 5. Experimental results from the application of the first heuristic in $P_{\text {param }}, P_{n o n-p a r a m}, P_{1-2 w}, P_{1-3 w}$ and $P_{1-4 w}$.

Heuristic 2

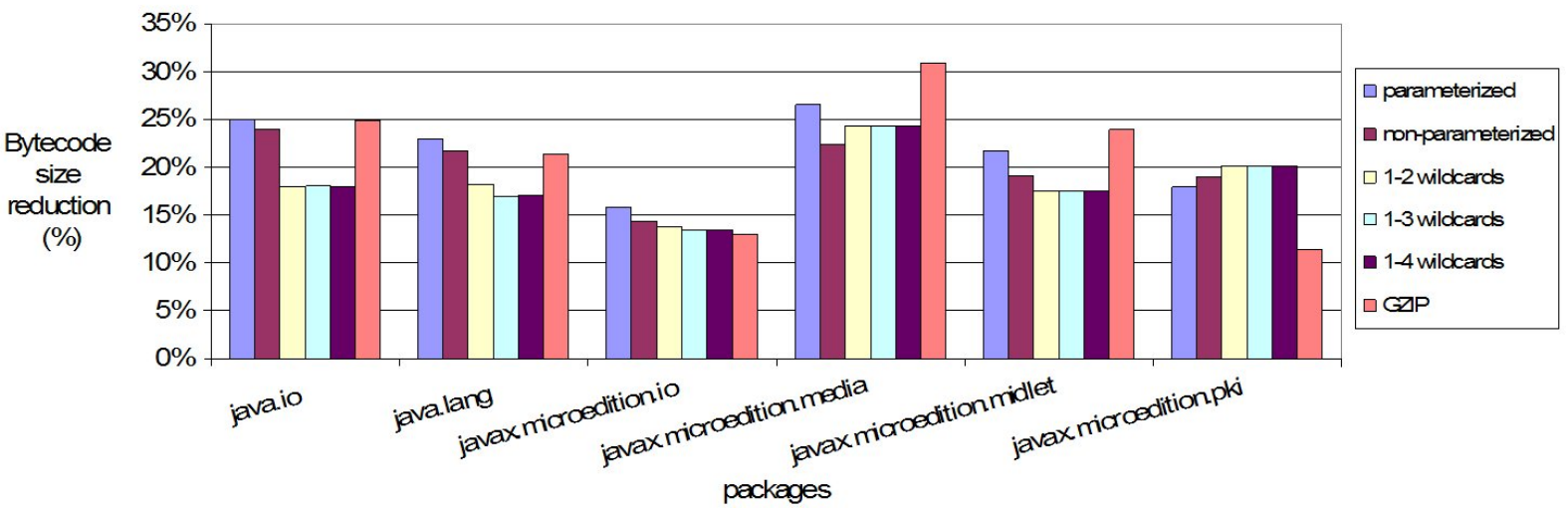

(a) Percentage of bytecode size reduction per MIDP package.

\begin{tabular}{lccccc}
\hline \multicolumn{1}{c}{ MIDP Package } & Parameterized & $\begin{array}{c}\text { Non- } \\
\text { parameterized }\end{array}$ & $\begin{array}{c}\text { Parameterized } \\
\text { patterns with } \\
\text { 1-2 widcards }\end{array}$ & $\begin{array}{c}\text { Parameterized } \\
\text { patterns with } \\
\text { 1-3 widcards }\end{array}$ & $\begin{array}{c}\text { Parameterized } \\
\text { patterns with } \\
\text { 1-4 widcards }\end{array}$ \\
\hline java.io & 2126.65 & 487.64 & 119.46 & 231.75 & 295.83 \\
\hline java.lang & 12731.71 & 4122.76 & 227.48 & 1417.55 & 1527.37 \\
\hline javax.microedition.io & 119.94 & 22.24 & 28.71 & 28.87 & 35.32 \\
\hline javax.microedition.media & 0.27 & 0.18 & 22.27 & 22.5 & 36.66 \\
\hline javax.microedition.midlet & 0.12 & 0.02 & 0.02 & 0.02 & 0.02 \\
\hline javax.microedition.pki & 0.01 & 0.01 & 0.33 & 0.42 & 0.45 \\
\hline
\end{tabular}

(b) Time to combine patterns per MIDP package (sec).

Fig. 6. Experimental results from the application of the second heuristic in $P_{\text {param }}, P_{n o n-p a r a m}, P_{1-2 w}, P_{1-3 w}$ and $P_{1-4 w}$.

11. In the case of the first heuristic, time increases from $26 \%$ to $34 \%$ for the different MIDP packages. Similarly, in the second heuristic time increases up to $34 \%$. With the exception of one package (javax.microedition.pki), the bytecode size reduction decreases. In the first heuristic the decrement ranges from $12 \%$ (Figure 8(a) third column - javax.microedition.media) to $-42 \%$ (Figure 8(a) second column - java.lang), while in the second heuristic the decrement ranges from $-7 \%$ (Figure $8(\mathrm{~b})$ 
javaio

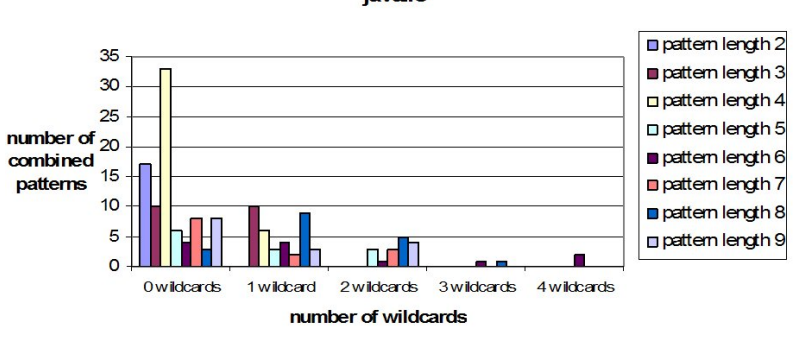

(a) java.io

javax.microeditionio

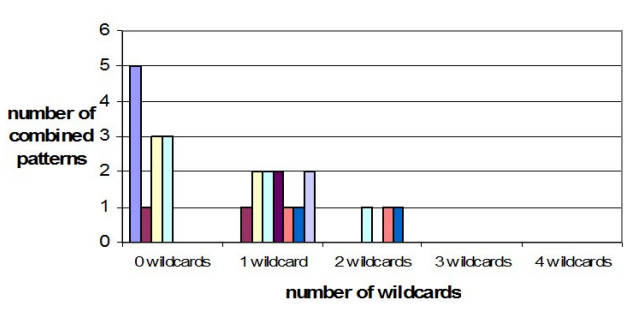

(c) javax.microedition.io

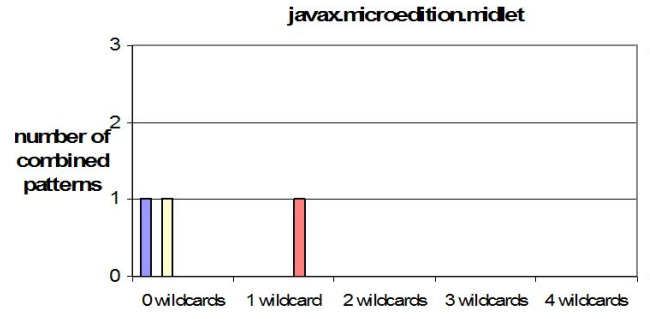

number of wildcards

(e) javax.microedition.midlet

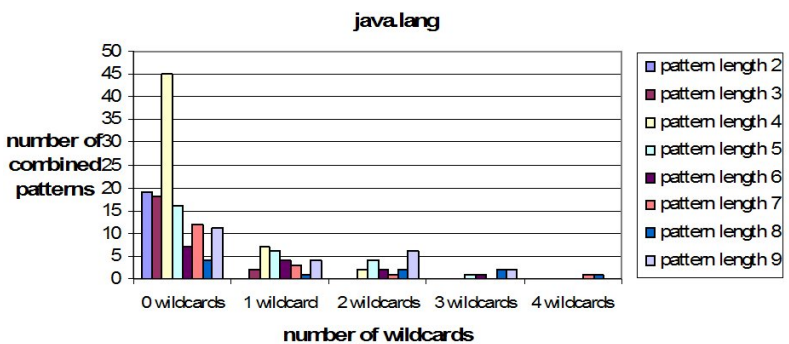

(b) java.lang

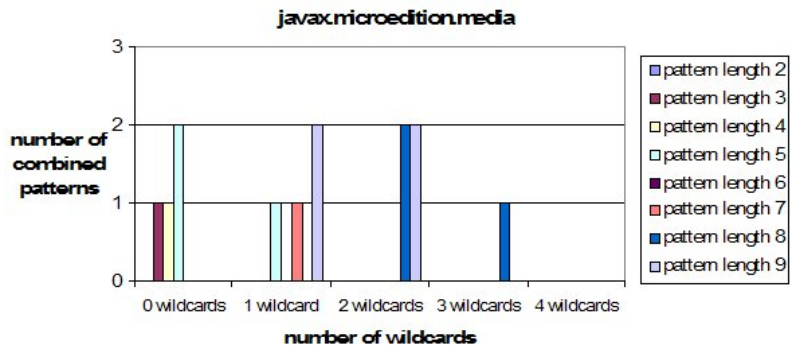

(d) javax.microedition.media javarmicroedition pli

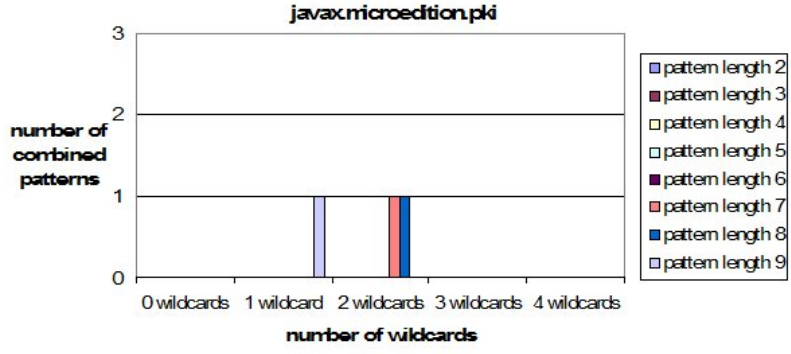

(f) javax.microedition.pki

Fig. 7. Patterns combined by the second heuristic in the case of Param.

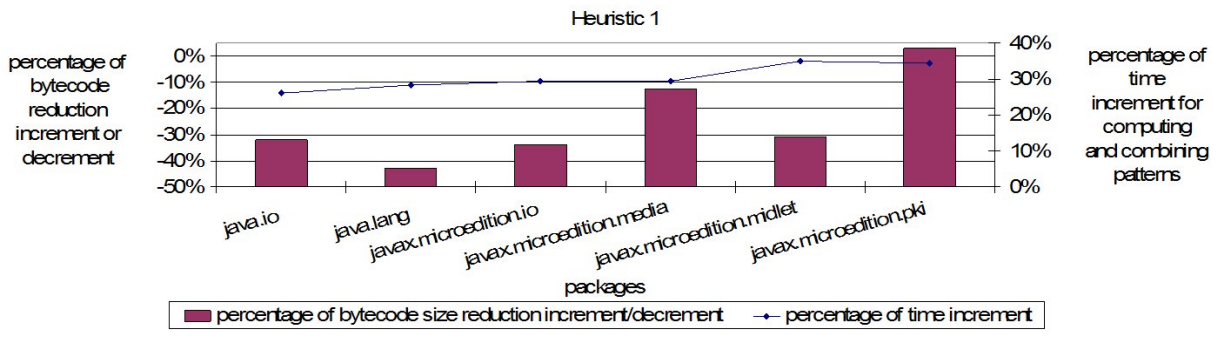

(a) Results obtained from the first heuristic.

Heurstic 2

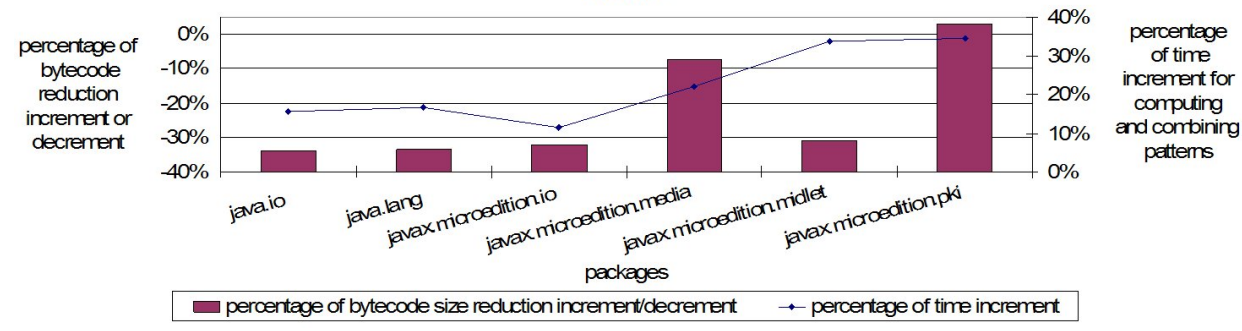

(b) Results obtained from the second heuristic.

Fig. 8. The impact of increasing the maximum length of the patterns in the bytecode size reduction obtained and the time required for discovering and combining the patterns.

third column - javax.microedition.media) to -33\% (Figure 8(b) first column - java.io). In the javax.microedition.pki package, the bytecode reduction increases up to $3 \%$ for both the heuristics (sixth column - Figures 8(a) and (b)).

Summarizing the results from our second set of experiments, we can conclude that very long patterns may not prove bene- 
ficial for the bytecode compression process. The best strategy is to start from discovering patterns whose maximum length is less or equal to 10 since the bytes required for encoding them in the dictionary is small. The discovery of longer patterns could be tried towards optimizing the compression, while keeping in mind that it may lead to worst results.

\section{B. Bytecode Decompression}

The decompression overhead introduced in the execution of applications that were compressed into interpretable bytecode is always an issue for the assessment of the compression technique that was used. However, this overhead depends on several factors concerning the application itself and the environment within which the application executes. Specifically, in the case of the technique examined in this paper, the overhead depends on the number of pattern instances encountered during the bytecode execution, the length of the patterns and the number of wildcards of these patterns. Moreover, the overhead depends on the characteristics of the particular device and the JVM used for the execution of the bytecode. It should be noted that the JVM may be even implemented as part of the processor used. The previous particularly holds in the case of Java processors such as picoJava ${ }^{5}$, Komodo ${ }^{6}$, aJile GEMCore ${ }^{7}$, $\mathrm{JOP}^{8}$ and several others. Given the previous remarks, in this paper we focus on examining the general behavior of the decompression overhead. To this end, we used randomly generated synthetic bytecode and a simple JVM main loop [25] that interprets the synthetic bytecode. The simple JVM main loop was realized for the purpose our experiments along with a random bytecode generator and the two decompression modules discussed in Section III C.

The random bytecode generator accepts as input a required percentage of bytecode size reduction and produces a synthetic bytecode sequence that can be compressed according to this percentage of bytecode size reduction. The generator further accepts as input the main features of a set of patterns that is generated with respect to the given features. This set of patterns is then used by the generator towards the construction of the target bytecode sequence, which consists of instances of the generated patterns, complemented with bytecode instructions that will not be compressed. Specifically, the main features that characterize a generated set of patterns are:

- The maximum length, $K$, of the patterns. Based on this feature, the generator creates patterns, whose length is uniformly distributed in the range $[2, K]$.

- The maximum number of wildcards, $M$, contained in the patterns. Given this feature, the generator builds patterns, whose number of wildcards is uniformly distributed in the range $[0, M]$.

- The maximum the number of pattern occurrences, $L$. According to this feature, the generator constructs a number of pattern occurrences in the target bytecode, which is uniformly distributed in the range $[2, L]$

\footnotetext{
${ }^{5} \mathrm{http} / / /$ www.sun.com/microelectronics/picoJava/

${ }^{6} \mathrm{http}: / /$ ipr.ira.uka.de/komodo/komodoEng.html

${ }^{7}$ http://www.ajile.com/products/jemcore.htm

${ }^{8} \mathrm{http}: / /$ www.jopdesign.com/
}

- A two-dimensional $K \times M$ matrix $F R$. The value of each matrix element $F R[k, m]$ (such that $k: 2, \ldots, K$ and $m$ : $0, \ldots, M)$ corresponds to the probability of generating a pattern of length $k$ with $m$ wildcards.

The JVM loop does not provide any advanced JIT compilation capabilities. Moreover, several typical JVM activities (e.g. verification, resolution, access control [25]) that precede the bytecode interpretation are omitted. Consequently, the overhead introduced by the decompression modules is expected to be high. In any case, the results are based on randomly generated bytecode and may not be representative of real applications that do not comply with the features used for the generated bytecode in our experiments. To perform our experiments we used 2 different environments. The first one relies on a $2 \mathrm{GHz}$ AMD Athlon XP $2400+$ with $256 \mathrm{~Kb}$ L2 cache, while the second one is based on a $500 \mathrm{MHz}$ UltraSPARC-IIe with $256 \mathrm{~Kb}$ L2 cache.

The input parameters of the generator relied on the results we obtained from the MIDP case study. In particular, we constructed 4 different sets of bytecode sequences, whose size was reduced $5 \%, 10 \%, 15 \%$ and $20 \%$, respectively. The compression of these bytecode sequences relied on patterns, which contained up to 4 wildcards. Similarly, we generated 4 more sets of bytecode sequences, whose size was reduced $5 \%$, $10 \%, 15 \%$ and $20 \%$, with respect to sets of patterns, which did not contain wildcards. In all cases, the maximum length of the patterns was set to 9 . The range of the pattern instances was $[2,12]$. The matrix $F R$ that was given as input to the generator was calculated with respect to the overall set of patterns that were combined using the second heuristic in the case of MIDP (Figure 7). The specific values of $F R$ that we used are given in Figure 9(a).

Figure 9(b) gives the average decompression overhead we obtained for the aforementioned sets of bytecode sequences. As expected, the overhead is quite high (however, it is close to the overhead reported in [4] for the CaffeineMark synthetic Java programs). The overhead linearly increases with the percentage of the bytecode size reduction. In general, the overhead in the case of the bytecode sequences that were compressed using patterns that did not contain wildcards was smaller, compared to the overhead in the case of the bytecode sequences that were compressed using patterns that contained wildcards. To further elaborate on the decompression overhead we measured the absolute time required for decompressing parameterized and non-parameterized patterns, whose length ranged from 2 to 9 bytes. The parameterized patterns comprised up to 3 wildcards. Specifically, the patterns of length 2 and 3 comprised 1 wildcard. The patterns of length 4,5 , and 6 comprised 2 wildcards. Finally, the patterns of length 7, 8 and 9 comprised 3 wildcards. The results we obtained are given in Figure 9(c). The time to decompress linearly increases with the length of the patterns.

\section{CONCLUSiON}

In this paper, we introduced a first approach that aimed at assessing the use of statistical pattern discovery for dictionarybased Java bytecode compression. In particular, we focused on 


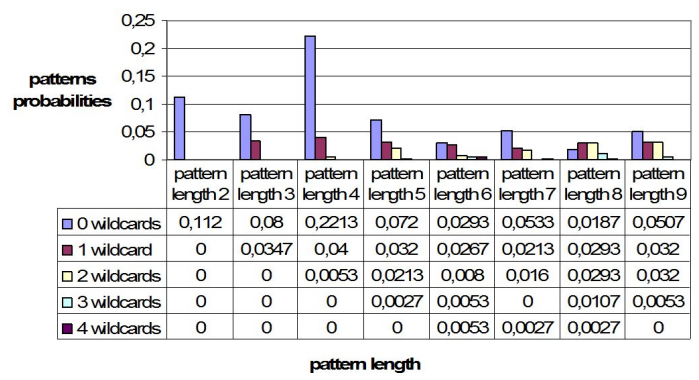

(a) Probabilities of generating patterns of length $k: 2, \ldots, 9$ with $m: 0, \ldots, 4$ wildcards.

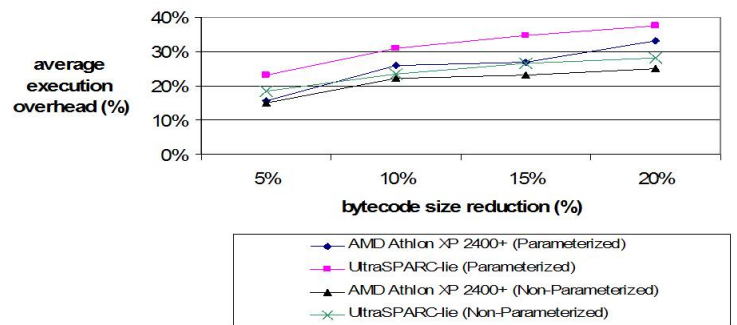

(b) Decompression overhead for the synthetic bytecode sequences.

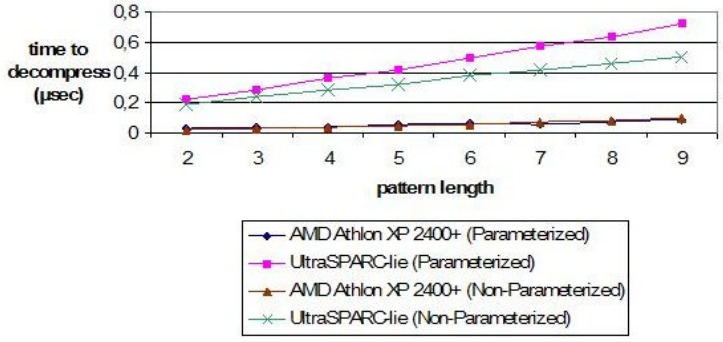

(c) Absolute times for pattern decompression (usec).

Fig. 9. Evaluating the decompression overhead of the parameterized pattern discovery technique.

the use of agglomerative clustering, a well-known hierarchical pattern discovery technique. The main outcome revealed from our assessment is that the examined technique promotes the identification of a rich collection of parameterized and non-parameterized patterns of variable lengths, which give the opportunity for obtaining good bytecode size reduction. However, the discovery of such a rich set of patterns for a given bytecode is certainly time consuming. Moreover, the complexity of finding useful combinations of patterns out of this set that result in a good bytecode size reduction is also high. To deal with the complexity of combining patterns we investigated two heuristics. Our experimental results showed that the length of the patterns should be appropriately customized so that it does not negatively affect the compression by requiring a large number of bytes for encoding the patterns. Moreover, our experimental results showed that non-parameterized patterns and patterns that contain a relatively small number of wildcards are the most useful in our case study. However, this observation may not hold for any possible Java bytecode. Based on these remarks, a good strategy for balancing the tradeoff between the time spent for discovering/combining patterns and the resulted bytecode size reduction is to apply the patterns discovery technique in an incremental manner. In a first step, the algorithm can be customized towards the discovery of small sets of patterns that contain few wildcards. Following, the small sets of patterns may serve as input to the algorithm towards the discovery of patterns that contain more wildcards, which may further improve the bytecode size reduction.

The incremental use of the agglomerative clustering algorithm is an interesting issue for further research, along with techniques that would allow pruning patterns that are not useful, early in the patterns discovery process. Currently, our work is also targeted towards further improving the efficiency of the pattern combination procedure. To this end, we aim at formulating the problem of pattern combination as a global optimization problem. This would allow us to investigate the use of classical global optimization techniques such as simulated annealing in conjunction with the proposed parameterized pattern discovery technique. Our future research further aims at the exploration of other, possibly more efficient, statistical methods for discovering patterns in Java bytecode.

\section{APPENDIX I \\ FURTHER RESULTS FROM THE MIDP CASE STUDY}

In this section, we provide further details regarding the experiments performed for the assessment of the parameterized pattern discovery technique discussed in this paper. Specifically, Figures 10 and 11, provide details regarding the bytecode size reduction obtained per different MIDP class from the application of the first and the second heuristics in $P_{\text {param }}$, $P_{\text {non-param }}, P_{1 w}, P_{2 w}, P_{3 w}$ and $P_{4 w}$. Similarly, Figures 12 and 13 give the bytecode size reduction obtained per different MIDP class from the application of the first and the second heuristics in $P_{\text {param }}, P_{n o n-p a r a m}, P_{1-2 w}, P_{1-3 w}$ and $P_{1-4 w}$. Finally, Figures 14 and 15 detail the impact of increasing the maximum length of the patterns found in MIDP from 9 to 11, in the bytecode size reduction obtained and the time required for compressing each MIDP class.

\section{REFERENCES}

[1] A. Beszédes, R. Ferenc, T. Gyimóthy, A. Dolenc, and K. Karsisto, "Survey of Code-Size Reduction Methods," ACM Computing Surveys, vol. 35, no. 3, pp. 223-267, 2003.

[2] D. Rayside, E. Mamas, and E. Hons, "Compact Java Binaries for Embedded Systems," in Proceedings of the 1999 ACM Conference of the Centre for Advanced Studies on Collaborative Research (CASCON'99), 1999, pp. 1-14.

[3] M. Latendresse and M. Feeley, "Generation of Fast Interpreters for Huffman Compressed Bytecode," Science of Computer Programming (Advances in Interpreters, Virtual Machines and Emulators), vol. 57, no. 3, pp. 295-317, 2005. 
[4] L. R. Clausen, U. P. Schultz, C. Consel, and G. Muller, "Java Bytecode Compression for Low-End Embedded Systems," ACM Transactions on Programming Languages and Systems, vol. 22, no. 3, pp. 471-489, 2000.

[5] M. Meilă and D. Hecherman, "An experimental comparison of modelbased clustering methods," Machine Learning, vol. 42, pp. 9-29, 2001.

[6] K. Blekas and A. Likas, "Incremental Mixture Learning for Clustering Discrete Data," in Lecture Notes in Artificial Intelligence, vol. 3025. Springer-Verlag, 2004, pp. 210-219.

[7] J. Ernst, W. Evans, C. W. Fraser, S. Lucco, and T. A. Proebsting, "Code Compression," in Proceedings of the ACM SIGPLAN Conference on Programming Language Design and Implementation (PLDI'97), 1997, pp. 358-365.

[8] Q. Brandly, R. N. Horspool, and J. Viter, "JAZZ: An Efficient Compressed Format for Java Archive Files," in Lecture Notes in Artificial Intelligence, vol. 3025. Springer-Verlag, 2004, pp. 210-219.

[9] M. Franz and T. Kistler, "Slim Binaries," Communications of the ACM, vol. 40, no. 12, pp. 87-94, 1997.

[10] W. Pugh, "Compressing Java Class Files," in Proceedings of the ACM SIGPLAN Conference on Programming Language Design and Implementation (PLDI'99), 1999, pp. 247-258.

[11] F. Tip, P. Sweeney, C. Laffra, A. Eisma, and D. Streeter, "Practical Extraction Techniques for Java," ACM Transactions on Programming Languages and Systems, vol. 24, no. 6, pp. 625-666, 2002.

[12] A. Wolfe and A. Chanin, "Executing Compressed Programs on an Embedded RISC Architecture," in Proceedings of the 25th IEEE International Symposium on Microarchitecture (Micro-25), 1992, pp. 81-92.

[13] M. Kozuch and A. Wolfe, "Compression of Embedded System Programs," in Proceedings of the International IEEE Conference on Computer Design: VLSI in Computers \& Processors, 1994, pp. 270-277.

[14] H. Lekatsas and A. Wolfe, "SAMC: A Code Compression Algorithm for Embedded Processors," IEEE Transactions on Computer Aided Design, vol. 18 , no. 12, pp. 1689-1701, 1999.

[15] K. D. Cooper and N. McIntosh, "Enhanced Code Compression for Embedded Risc Processors," ACM SIGPLAN Notices, vol. 5, pp. 139149,1999

[16] IBM, "CodePack: PowerPC Code Compression Utility User's Manual v3.0," IBM Corporation, Tech. Rep., 1998

[17] C. R. Lefurgy, P. L. Bird, I-C. Chen and T. N. Mudge, "Improving Code Density Using Compression Techniques," in Proceedings of the 30th ACM/IEEE International Symposium on Microarchitecture (Micro30), 1997, pp. 194-203.

[18] S. K. Debray, W. Evans, R. Muth and B. De Sutter, "Compiler Techniques for Code Compaction," ACM Transactions on Programming Languages and Systems, vol. 22, no. 2, pp. 378-415, 2000.

[19] B. De Sutter, B. De Bus and K. De Bosschere, "Sifting out the Mud: Low Level C++ Code Reuse," in Proceedings of the 17th ACM SIGPLAN Conference on Object-Oriented Programming Systems Languages and Applications (OOPSLA'02), 2002, pp. 275-291.

[20] J. Krinke, "Identifying Similar Code with Program Dependence Graphs," in Proceedings of the 8th IEEE Working Conference on Reverse Engineering, 2001, pp. 301-309.

[21] W. Cheung, W. Evans, and J. Moses, Software and Compilers for Embedded Systems: 7th International Workshop (SCOPES'03), ser. LNCS. Springer-Verlag, 2003, vol. 2826, ch. Predicated Instructions for Code Compaction, pp. 17-31.

[22] W. Evans and C. Fraser, "Bytecode Compression via Profiled Grammar Rewriting," in Proceedings of the ACM SIGPLAN Conference on Programming Language Design and Implementation (PLDI'01), 2001, pp. $148-155$.

[23] R. Komondoor and S. Horwitz, "Effective, Automatic Procedure Extraction," in Proceedings of the 11th IEEE International Workshop on Program Comprehension (IWPC'03), 2003, pp. 33-43.

[24] Y. Bengio and S. Bengio, "Modeling High-Dimensional Discrete Data with Multi-Layer Neural Networks," in Advances in Neural Processing Systems 12, S. Solla, T. Leen, and K.-R. Móller, Eds. MIT Press, 2000, pp. 400-406.

[25] T. Lindholm and F. Yellin, The Java Virtual Machine Specification. SUN Microsystems, 1999, ch. 5: The Structure of the Java Virtual Machine.

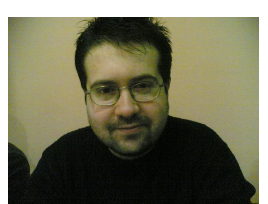

Dimitris Saougkos received his B.Sc. in 2004 from the Department of
Computer Science of the University of Ioannina, Greece. Following, he obtained his M.Sc. in 2006 from the same department. His main research interests include code compression, compilers, programming languages, middleware,

mobile and embedded computing systems.
George Manis is a Lecturer in the Department of Computer Science of the University of Ioannina, Greece. He received his Diploma in Electrical and Computer Engineering in 1992 from the National Technical University of Athens (NTUA), Greece. In 1993, he obtained his MSc from the Queen Mary and Westfield College (QMW), University of London. In 1997, he obtained his Ph.D. in Computer Engineering from NTUA. His main research interests include computing systems and architectures, compilers, and biomedical engineering.

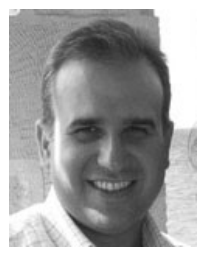

Konstantinos Blekas received his Diploma in Electrical Engineering in 1993 and his Ph.D. in Electrical and Computer Engineering in 1997, both from the National Technical University of Athens (NTUA). Since 2002, he has been with the Department of Computer Science, University of Ioannina, Greece, where he is currently a Lecturer. His research interests include artificial intelligence, statistical pattern recognition, machine learning, and bioinformatics.

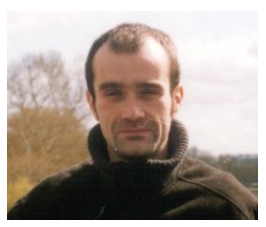

Apostolos V. Zarras holds a Lecturer position at the Department of Computer Science, University of Ioannina, Greece. He received his B.Sc. in Computer Science in 1994 from the Department of Computer Science, University of Crete. In 1996, he received his M.Sc. in Distributed Systems and Computer Architecture, from the same department. He obtained his Ph.D in Distributed Systems and Software Architectures in 2000 from the University of Rennes I, France. From 1999 to 2001 Apostolos Zarras worked as a researcher at the ARLES Group of INRIA-Rocquencourt. His research interests include middleware, modeldriven architecture development and pervasive computing. 


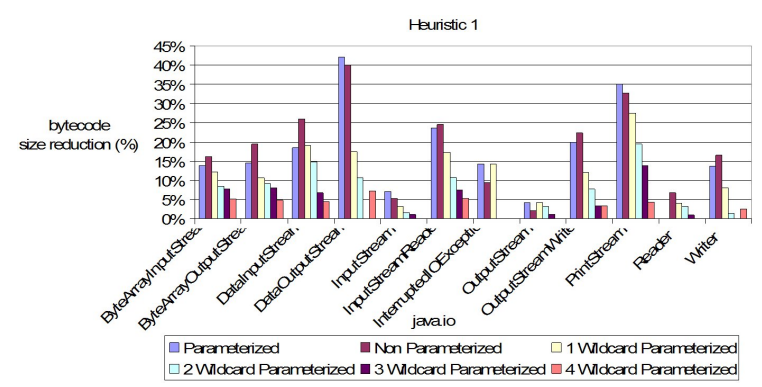

(a) java.io

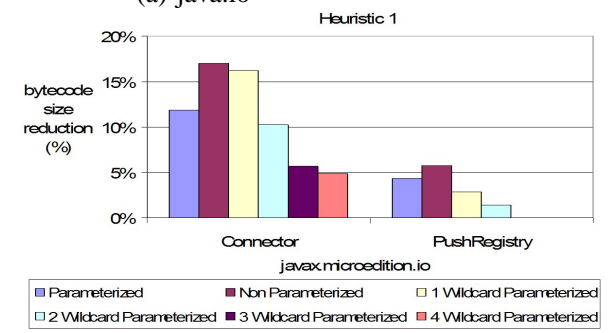

(c) javax.microedition.io

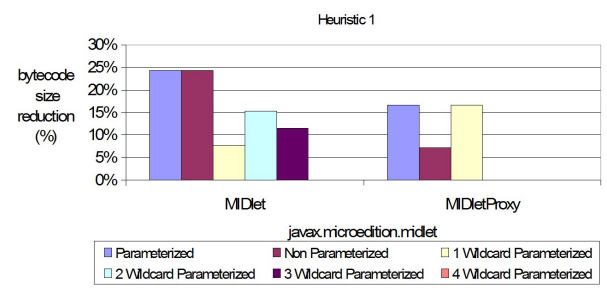

(e) javax.microedition.midlet

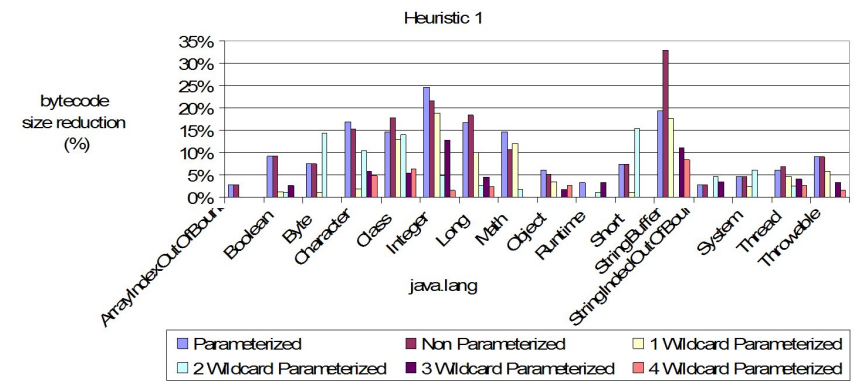

(b) java.lang

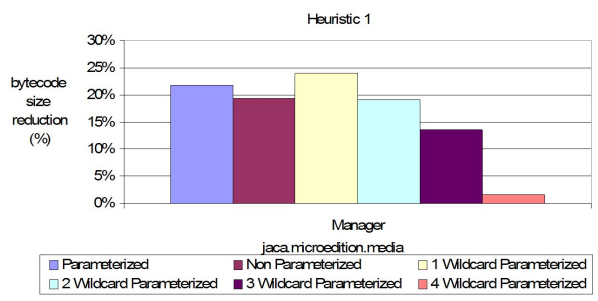

(d) javax.microedition.media

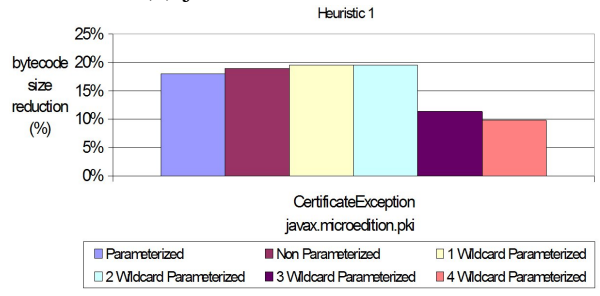

(f) javax.microedition.pki

Fig. 10. Experimental results - per MIDP class - from the application of the first heuristic in $P_{\text {param }}, P_{n o n-p a r a m}, P_{1 w}, P_{2 w}, P_{3 w}$ and $P_{4 w}$.

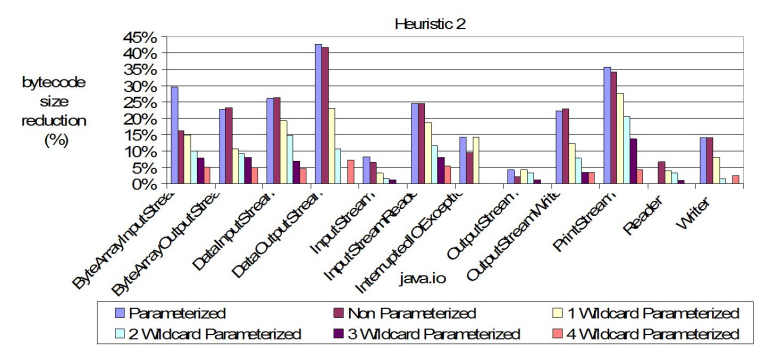

(a) java.io

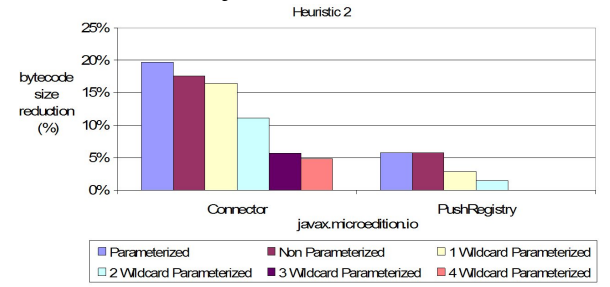

(c) javax.microedition.io

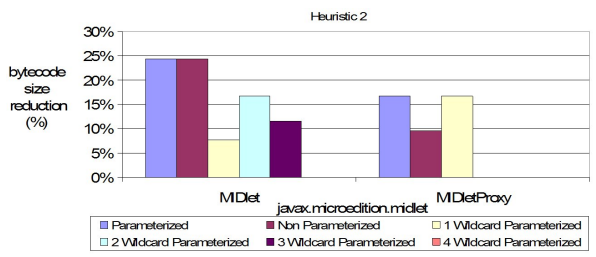

(e) javax.microedition.midlet

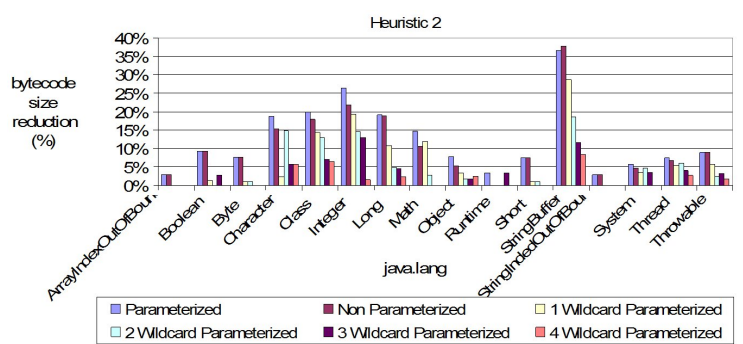

(b) java.lang

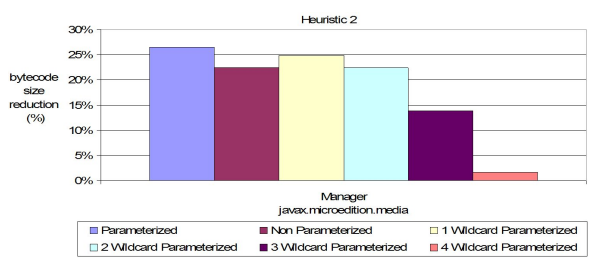

(d) javax.microedition.media

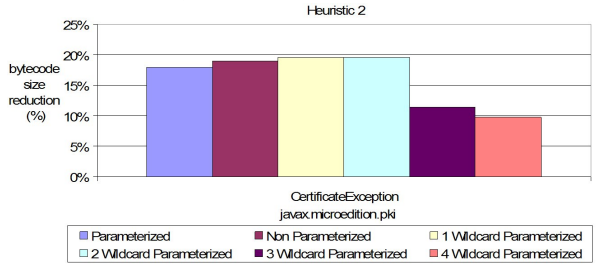

(f) javax.microedition.pki

Fig. 11. Experimental results - per MIDP class - from the application of the second heuristic in $P_{\text {param }}, P_{n o n-\text { param }}, P_{1 w}, P_{2 w}, P_{3 w}$ and $P_{4 w}$. 


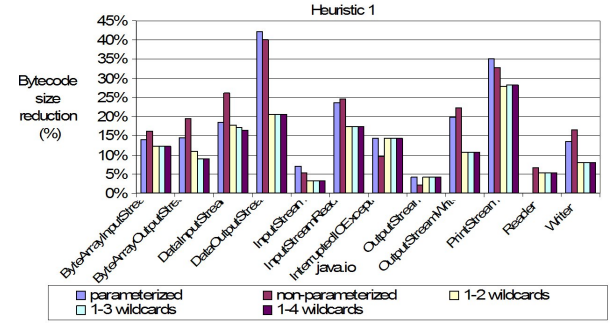

(a) java.io

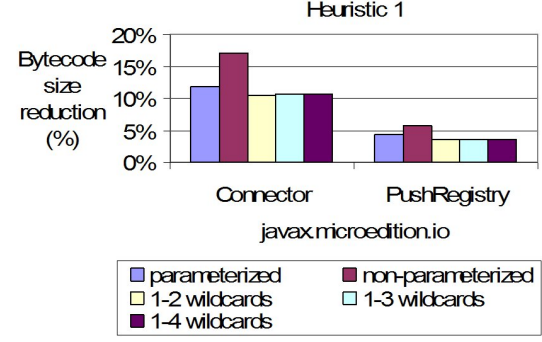

(c) javax.microedition.io

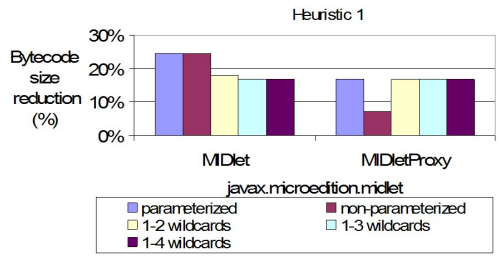

(e) javax.microedition.midlet

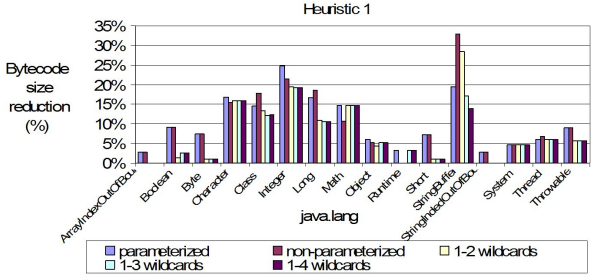

(b) java.lang

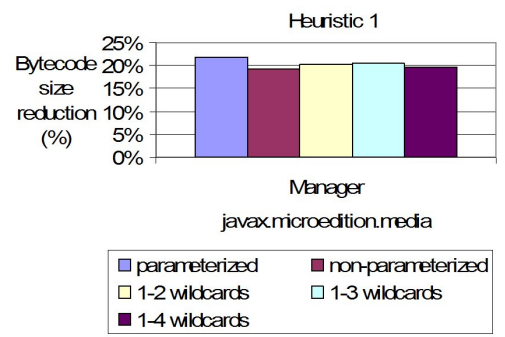

(d) javax.microedition.media

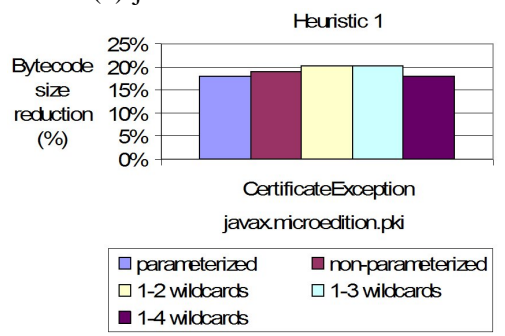

(f) javax.microedition.pki

Fig. 12. Experimental results - per MIDP class - from the application of the first heuristic in $P_{\text {param }}, P_{n o n-p a r a m}, P_{1-2 w}, P_{1-3 w}$ and $P_{1-4 w}$.

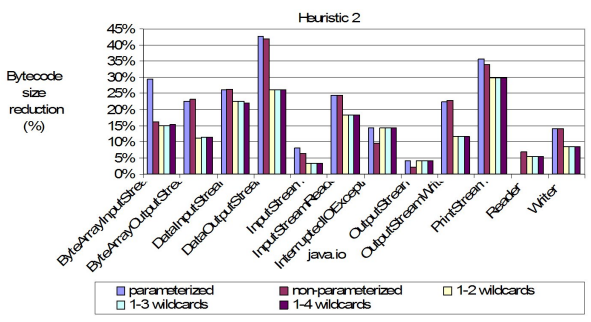

(a) java.io

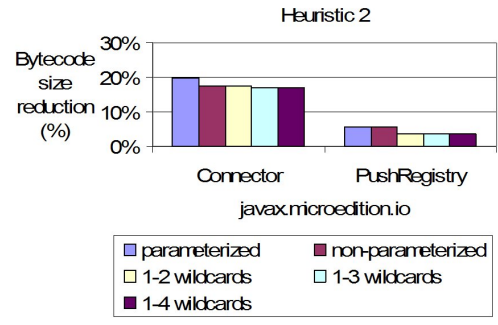

(c) javax.microedition.io

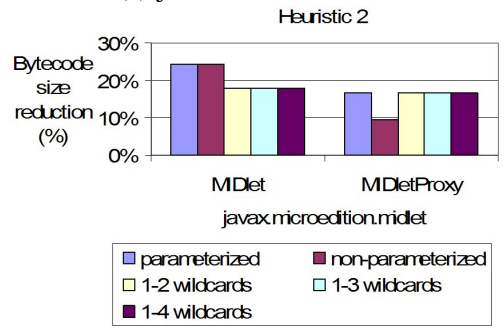

(e) javax.microedition.midlet

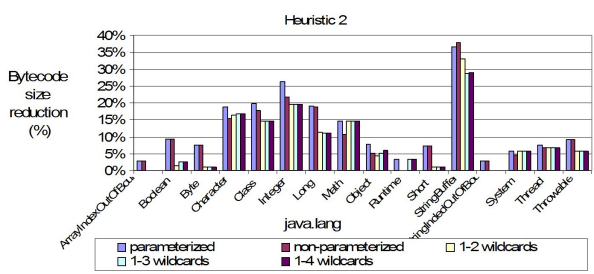

(b) java.lang Heurstic 2

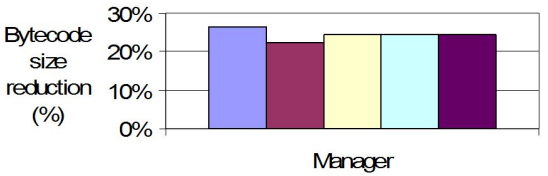

javaxmicroedition.media

\begin{tabular}{|ll|}
\hline$\square$ parameterized & $\square$ non-parameterized \\
$\square 1-2$ vildcards & $\square 1-3$ vildcards \\
$\square 1-4$ vildcards &
\end{tabular}

(d) javax.microedition.media Heuristic 2

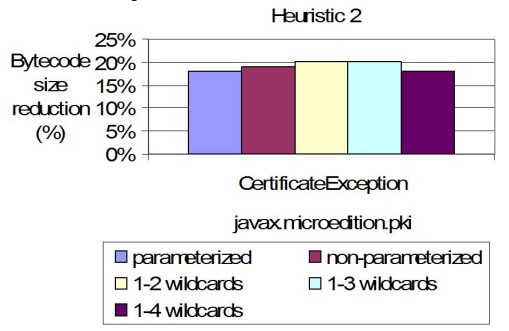

(f) javax.microedition.pki

Fig. 13. Experimental results - per MIDP class - from the application of the second heuristic in $P_{\text {param }}, P_{n o n-p a r a m}, P_{1-2 w}, P_{1-3 w}$ and $P_{1-4 w}$. 


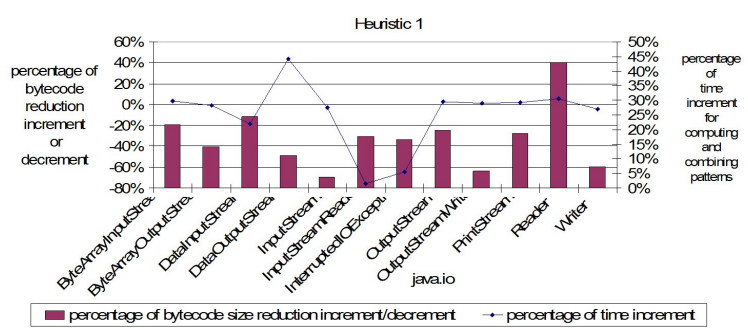

(a) java.io

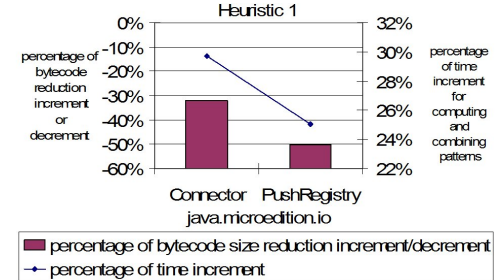

(c) javax.microedition.io

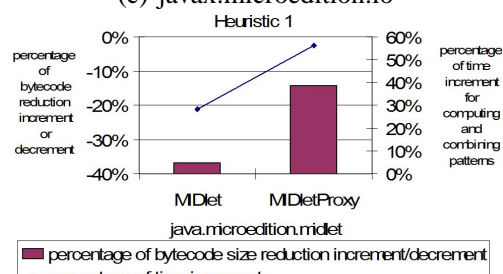
$\rightarrow$ percentage of time increment

(e) javax.microedition.midlet

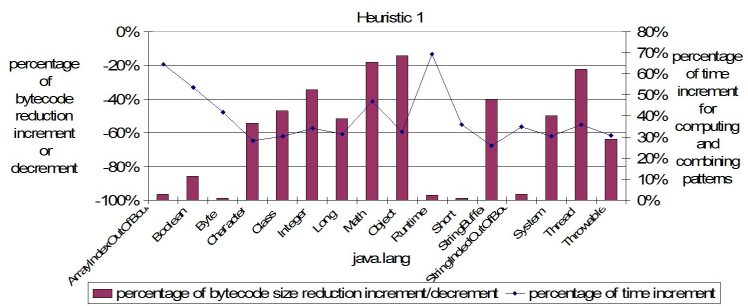

(b) java.lang

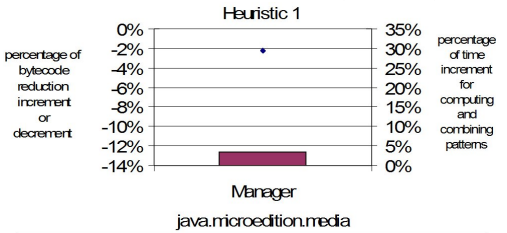

- percentage of bytecode size reduction increment/decrement $\rightarrow$ percentage of time increment

(d) javax.microedition.media

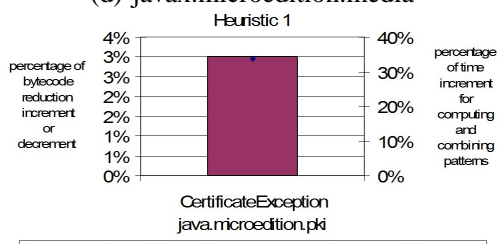

percentage of bytecode size reduction increment/decrement $\rightarrow$ percentage of time increment

(f) javax.microedition.pki

Fig. 14. The impact - per MIDP class - of increasing the maximum length of the patterns in the bytecode size reduction obtained and the time required for discovering and combining the patterns (first heuristic).

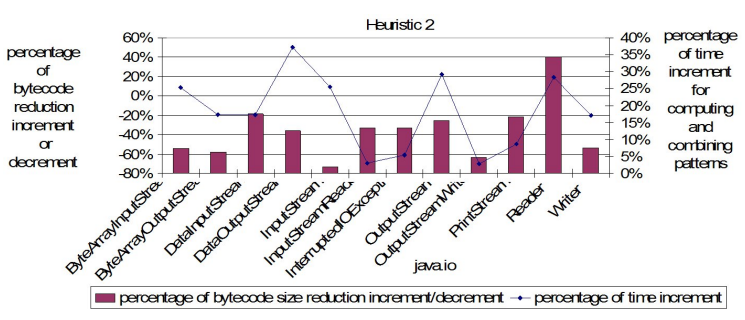

(a) java.io

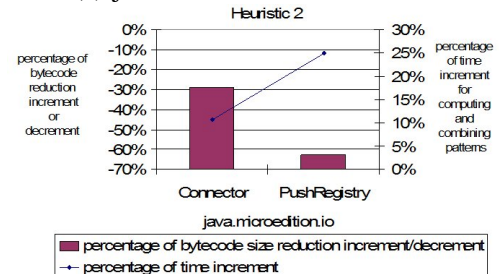

(c) javax.microedition.io

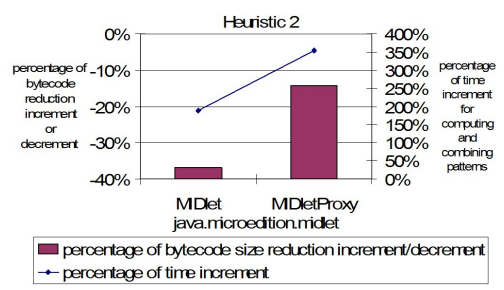

(e) javax.microedition.midlet

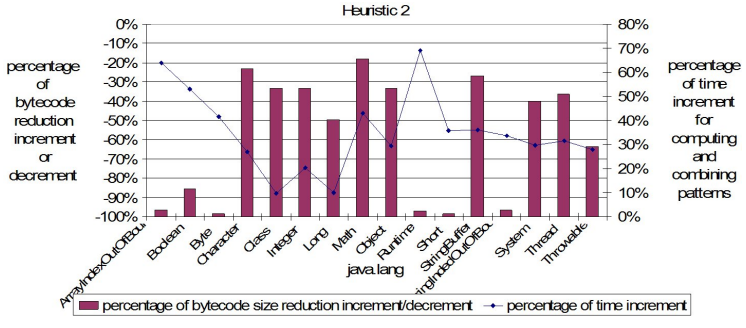

(b) java.lang

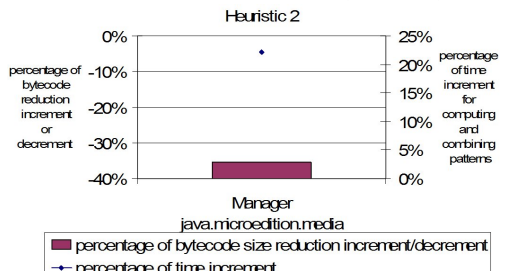

(d) javax.microedition.media

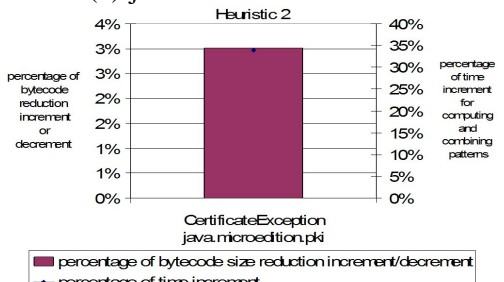

- percentage of time incremert

(f) javax.microedition.pki

Fig. 15. The impact - per MIDP class - of increasing the maximum length of the patterns in the bytecode size reduction obtained and the time required for discovering and combining the patterns (second heuristic). 